\title{
Weyl Law on Asymptotically Euclidean Manifolds
}

\author{
Sandro Coriasco® and Moritz Dollø
}

\begin{abstract}
We study the asymptotic behaviour of the eigenvalue counting function for self-adjoint elliptic linear operators defined through classical weighted symbols of order $(1,1)$, on an asymptotically Euclidean manifold. We first prove a two-term Weyl formula, improving previously known remainder estimates. Subsequently, we show that under a geometric assumption on the Hamiltonian flow at infinity, there is a refined Weyl asymptotics with three terms. The proof of the theorem uses a careful analysis of the flow behaviour in the corner component of the boundary of the double compactification of the cotangent bundle. Finally, we illustrate the results by analysing the operator $Q=\left(1+|x|^{2}\right)(1-\Delta)$ on $\mathbb{R}^{d}$.
\end{abstract}

Mathematics Subject Classification. Primary 58J50; Secondary 58J40, $35 \mathrm{P} 20$.

\section{Introduction}

Let $(X, g)$ be a $d$-dimensional asymptotically Euclidean manifold. More explicitly, $X$ belongs to a class of compact manifolds with boundary, whose interior is equipped with a Riemannian metric $g$ which assumes a specific form close to the boundary $\partial X$ (see Definition 29 in Section A.1 of "Appendix"). The elements of such class are also known as scattering manifolds, asymptotically conic manifolds, or manifolds with large conic ends. A typical example is the unit ball $\mathbb{B}^{d}$, equipped with a scattering metric.

On $X$, we consider a self-adjoint positive operator $P$, elliptic in the SGcalculus of order $(m, n)$ with $m, n \in(0, \infty) .{ }^{1}$ By the compact embedding of

\footnotetext{
${ }^{1}$ We refer to Sect. 2, (4) and (5) for the precise definitions.
} 
weighted Sobolev spaces, the resolvent is compact, and hence, the spectrum of $P$ consists of a sequence of eigenvalues

$$
0<\lambda_{1} \leq \lambda_{2} \leq \cdots \rightarrow+\infty .
$$

The goal of this article is to study the Weyl law of $P$, that is, the asymptotics of its counting function,

$$
N(\lambda)=\#\left\{j: \lambda_{j}<\lambda\right\} .
$$

Hörmander [18] proved, for a positive elliptic self-adjoint classical pseudodifferential operator of order $m>0$ on a compact manifold, the Weyl law

$$
N(\lambda)=\gamma \cdot \lambda^{\frac{d}{m}}+O\left(\lambda^{\frac{d-1}{m}}\right), \quad \lambda \rightarrow+\infty .
$$

It was pointed out that, in general, this is the sharp remainder estimate, since the exponent of $\lambda$ in the remainder term cannot be improved for the Laplacian on the sphere. It was subsequently shown by Duistermaat and Guillemin [15] that, under a geometric assumption, there appears an additional term $\gamma^{\prime} \lambda^{(d-1) / m}$ and the remainder term becomes $o\left(\lambda^{(d-1) / m}\right)$.

In the case of SG-operators on manifolds with cylindrical ends (see Definition 41 and the relationship with asymptotically Euclidean manifolds at the end of Section A.4 of "Appendix"), the leading order of the Weyl asymptotics was found by Maniccia and Panarese [22]. Battisti and Coriasco [3] improved the remainder estimate to $O\left(\lambda^{d / \max \{m, n\}-\epsilon}\right)$ for some $\epsilon>0$. For $m \neq n$, Coriasco and Maniccia [10] proved the general sharp remainder estimate. We recall that $\mathbb{R}^{d}$ is the simplest example of manifold with one cylindrical end.

In Theorem 1, we prove the analogue of Hörmander's result for $m=n$. This provides a more precise remainder term compared to the earlier result given in [3]. If the geodesic flow at infinity generated by the corner component $p_{\psi e}$ of the principal symbol of $P$ is sufficiently generic, we have an even more refined estimate, parallel to the Duistermaat-Guillemin theorem, described in Theorem 2.

Theorem 1. Let $P \in \mathrm{OpSG}_{\mathrm{cl}}^{m, m}(X), m>0$, be a self-adjoint, positive, elliptic $\mathrm{SG}$-classical pseudodifferential operator on an asymptotically Euclidean manifold $X$, and $N(\lambda)$ its associated counting function. Then, the corresponding Weyl asymptotics reads as

$$
N(\lambda)=\gamma_{2} \lambda^{\frac{d}{m}} \log \lambda+\gamma_{1} \lambda^{\frac{d}{m}}+O\left(\lambda^{\frac{d-1}{m}} \log \lambda\right) .
$$

If $X^{o}$ is a manifold with cylindrical ends, then the coefficients $\gamma_{j}, j=1,2$, are given by

$$
\begin{aligned}
\gamma_{2} & =\frac{\operatorname{TR}\left(P^{-\frac{d}{m}}\right)}{m \cdot d}, \\
\gamma_{1} & =\widehat{\mathrm{TR}}_{x, \xi}\left(P^{-\frac{d}{m}}\right)-\frac{\mathrm{TR}\left(P^{-\frac{d}{m}}\right)}{d^{2}},
\end{aligned}
$$

where $\mathrm{TR}$ and $\widehat{\mathrm{TR}}_{x, \xi}$ are suitable trace operators on the algebra of $\mathrm{SG}$-operators on $X$. 
Theorem 2. Let $P \in \mathrm{OpSG}_{\mathrm{cl}}^{m, m}(X)$ and $N(\lambda)$ be as in Theorem 1. Denote by $p_{\psi e}$ the corner component of the principal symbol of $P$. If the set of periodic orbits of the Hamiltonian flow of $\mathrm{X}_{f}, f=\left(p_{\psi e}\right)^{\frac{1}{m}}$, has measure zero on $\mathcal{W}^{\psi e}$, then we have the estimate

$$
N(\lambda)=\gamma_{2} \lambda^{\frac{d}{m}} \log \lambda+\gamma_{1} \lambda^{\frac{d}{m}}+\gamma_{0} \lambda^{\frac{d-1}{m}} \log \lambda+o\left(\lambda^{\frac{d-1}{m}} \log \lambda\right),
$$

with the coefficients $\gamma_{2}$ and $\gamma_{1}$ given in Theorem 1, and

$$
\gamma_{0}=\frac{\operatorname{TR}\left(P^{-\frac{d-1}{m}}\right)}{m \cdot(d-1)},
$$

if $X^{o}$ is a manifold with cylindrical ends.

Remark 3. The trace operators $\mathrm{TR}$ and $\widehat{\mathrm{TR}}_{x, \xi}$ appearing in Theorems 1 and 2 were introduced in [3], see also Section A.3. in "Appendix". The coefficient $\gamma_{0}$ can be calculated as the Laurent coefficient of order -2 at $s=d-1$ of $\zeta(s)$, the spectral $\zeta$-function associated with $P$.

Remark 4. To our best knowledge, this is the first result of a logarithmic Weyl law with the remainder being one order lower than the leading term. (We refer, for example, to [2] for a discussion of other settings with logarithmic Weyl laws.)

Remark 5. In view of the analysis at the end of Section A.4 in "Appendix", we can apply our results to $\mathrm{SG}$-operators on the manifold $X=\mathbb{B}^{d}$, equipped with an arbitrary scattering metric $g$, that is, to SG-operators on the manifold with one cylindrical end $\mathbb{R}^{d}$, identified with $\left(\mathbb{B}^{d}\right)^{o}$ by radial compactification.

Next, we apply our results to the model operator $P$ associated with the symbol $p(x, \xi)=\langle x\rangle \cdot\langle\xi\rangle,\langle z\rangle=\sqrt{1+|z|^{2}}, z \in \mathbb{R}^{d}$, that is, $P=\langle\cdot\rangle \sqrt{1-\Delta}$. In particular, we observe that the condition on the underlying Hamiltonian flow in Theorem 2 is not satisfied and compute explicitly the coefficients $\gamma_{1}$ and $\gamma_{2}$.

Theorem 6. Let $P=\langle\cdot\rangle\langle D\rangle \in \mathrm{OpSG}_{\mathrm{cl}}^{1,1}\left(\mathbb{R}^{d}\right)$. Then,

$$
N(\lambda)=\gamma_{2} \lambda^{d} \log \lambda+\gamma_{1} \lambda^{d}+O\left(\lambda^{d-1} \log \lambda\right) .
$$

Here, the coefficients are

$$
\begin{aligned}
& \gamma_{2}=\frac{\left[\operatorname{vol}\left(\mathbb{S}^{d-1}\right)\right]^{2}}{(2 \pi)^{d}} \cdot \frac{1}{d} \\
& \gamma_{1}=-\frac{\left[\operatorname{vol}\left(\mathbb{S}^{d-1}\right)\right]^{2}}{(2 \pi)^{d}} \cdot \frac{1}{d} \cdot\left[\Psi\left(\frac{d}{2}\right)+\gamma+\frac{1}{d}\right],
\end{aligned}
$$

where $\gamma=\lim _{n \rightarrow+\infty}\left(\sum_{k=1}^{n} \frac{1}{k}-\log n\right)$ is the Euler-Mascheroni constant and

$$
\Psi(x)=\frac{d}{\mathrm{~d} x} \log \Gamma(x)
$$

is the digamma function. 
This implies that the Weyl asymptotics of the operator

$$
Q=\left(1+|x|^{2}\right)(1-\Delta)
$$

is given by

$$
N(\lambda)=\frac{\gamma_{2}}{2} \lambda^{\frac{d}{2}} \log \lambda+\gamma_{1} \lambda^{\frac{d}{2}}+O\left(\lambda^{\frac{d-1}{2}} \log \lambda\right),
$$

with the same coefficients given in Theorem 6 .

Remark \%. It could be conjectured that many operators satisfying the assumptions of Theorem 1 also satisfy the additional geometric requirement which allows to obtain the refined Weyl formula (2) (cf. [14] for a proof of such fact in a different setting). However, we remark that it is still an open problem to construct explicitly an operator fulfilling the hypotheses of Theorem 2 .

Remark 8. Operators like $Q$ arise, for instance, as local representations of Schrödinger-type operators of the form $H=-\Delta_{\mathfrak{h}}+V$ on manifolds with ends, for appropriate choices of the metric $\mathfrak{h}$ and potential $V$ (see Section A.4 in "Appendix" for a description of this class of manifolds adopted, e.g. in $[3,22])$. We just sketch an example of construction of such an operator (see [8, Example 5.21] for the details). Consider the cylinder $C=\{(u, v, z) \in$ $\left.\mathbb{R}^{3}: u^{2}+v^{2}=1, z>1\right\}=\mathbb{S}^{1} \times(1,+\infty) \subset \mathbb{R}^{3}$ as the model of an end. Pulling back to the metric $\mathfrak{h}$ on $C$ the metric $\mathfrak{h}^{\prime}$ on $\mathbb{R}^{3}$ given by $\mathfrak{h}^{\prime}=4^{-1} \operatorname{diag}\left(z^{2}\langle z\rangle^{-n}\right.$, $\left.z^{2}\langle z\rangle^{-n}, 4\langle z\rangle^{-n}\right), n>0$, it turns out that, in suitable local coordinates $x=\left(x_{1}, x_{2}\right) \in \mathbb{R}^{2}$ on $C$, the Laplace-Beltrami operator has the form

$$
\Delta_{\mathfrak{h}}=\left(1+x_{1}^{2}+x_{2}^{2}\right)^{\frac{n}{2}}\left(\partial_{x_{1}}^{2}+\partial_{x_{2}}^{2}\right)=\langle x\rangle^{n} \Delta,
$$

with $\Delta$ the standard Laplacian. Choosing then, in local coordinates, $V(x)=$ $\langle x\rangle^{n}$, we find

$$
H=-\Delta_{\mathfrak{h}}+V=\langle x\rangle^{n}(1-\Delta) .
$$

It is straightforward to see that $H \in \mathrm{OpSG}_{\mathrm{cl}}^{2, n}$ and it is elliptic (see Sect. 2), as claimed.

The proofs of Theorems 1 and 2 are broken into two parts. First, we will establish a connection between the wave trace near $t=0$ and the zeta function, to calculate the coefficients of the wave trace. Then, we use a parametrix construction to relate the wave trace to the counting function.

The paper is organized as follows. In Sect. 2, we fix most of the notation used throughout the paper and recall the basic elements of the calculus of SG-classical pseudodifferential operators, the associated wavefront set, and the computation of the parametrix of Cauchy problems for SG-hyperbolic operators of order $(1,1)$. In particular, we quickly recall the invariance properties of the SG-calculus. In Sect. 3, we consider the wave trace of a SG-classical operator $P$ of order $(1,1)$. Section 4 is devoted to study the relation between the wave trace and the spectral $\zeta$-function of $P$. In Sect. 5 , we prove our main Theorems 1 and 2, while in Sect. 6, we examine the example given by the model operator $P=\langle\cdot\rangle\langle D\rangle$ and prove Theorem 6 . For the convenience of 
the reader, we conclude with an "Appendix", including a few facts concerning asymptotically Euclidean manifolds and manifolds with cylindrical ends, including a comparison of the two notions at the end of Section A.4. We also give a short summary of the various trace operators and SG-Fourier integral operators.

\section{SG-Calculus}

The Fourier transform $\mathcal{F}: \mathcal{S}\left(\mathbb{R}^{d}\right) \rightarrow \mathcal{S}\left(\mathbb{R}^{d}\right)$ is defined by

$$
(\mathcal{F} u)(\xi)=\widehat{u}(\xi)=\int \mathrm{e}^{-i x \xi} u(x) \mathrm{d} x, u \in \mathcal{S}\left(\mathbb{R}^{d}\right),
$$

and extends by duality to a bounded linear operator $\mathcal{F}: \mathcal{S}^{\prime}\left(\mathbb{R}^{d}\right) \rightarrow \mathcal{S}^{\prime}\left(\mathbb{R}^{d}\right)$.

The set of pseudodifferential operators $A=a^{w}(x, D)=\mathrm{Op}^{w}(a)$ : $\mathcal{S}\left(\mathbb{R}^{d}\right) \rightarrow \mathcal{S}^{\prime}\left(\mathbb{R}^{d}\right)$ on $\mathbb{R}^{d}$ with Weyl symbol $a \in \mathcal{S}^{\prime}\left(\mathbb{R}^{2 d}\right)$ can be defined through the Weyl quantization ${ }^{2}$

$$
A u(x)=(2 \pi)^{-d} \iint \mathrm{e}^{i(x-y) \xi} a((x+y) / 2, \xi) u(y) \mathrm{d} y \mathrm{~d} \xi, \quad u \in \mathcal{S}\left(\mathbb{R}^{d}\right) .
$$

A smooth function $a \in \mathcal{C}^{\infty}\left(\mathbb{R}^{d} \times \mathbb{R}^{d}\right)$ is a SG-symbol of order $\left(m_{\psi}, m_{e}\right) \in$ $\mathbb{R}^{2}$, and we write $a \in \mathrm{SG}^{m_{\psi}, m_{e}}\left(\mathbb{R}^{2 d}\right)$, if for all multiindices $\alpha, \beta \in \mathbb{N}_{0}^{d}$, there exists $C_{\alpha \beta}>0$ such that, for all $x, \xi \in \mathbb{R}^{d}$,

$$
\left|\partial_{x}^{\alpha} \partial_{\xi}^{\beta} a(x, \xi)\right| \leq C_{\alpha \beta}\langle\xi\rangle^{m_{\psi}-|\beta|}\langle x\rangle^{m_{e}-|\alpha|} .
$$

The space $\mathrm{SG}^{m_{\psi}, m_{e}}\left(\mathbb{R}^{2 d}\right)$ becomes a Fréchet space with the seminorms being the best constants in (5). The space of all SG-pseudodifferential operators of order $\left(m_{\psi}, m_{e}\right)$ is denoted by

$$
\mathrm{OpSG} \mathrm{SG}^{m_{\psi}, m_{e}}\left(\mathbb{R}^{d}\right)=\left\{\mathrm{Op}^{w}(a): a \in \mathrm{SG}^{m_{\psi}, m_{e}}\left(\mathbb{R}^{2 d}\right)\right\}
$$

The corresponding calculus was established in the 70s by Cordes and Parenti (see, for example, [4,32]). The letter "G" in the notation, after the usual initial "S" for "symbol space", stands for "global". This calculus of symbols of product type, globally defined on $\mathbb{R}^{d}$, was also considered by Shubin (see [38]). Actually, the SG-calculus on $\mathbb{R}^{d}$ is a special case of the Weyl calculus (see [19, Sections 18.4-18.6]), associated with the slowly varying Riemannian metric on $\mathbb{R}^{2 d}$ given by

$$
g_{(y, \eta)}(x, \xi)=\langle y\rangle^{-2}|x|^{2}+\langle\eta\rangle^{-2}|\xi|^{2}
$$

(see, for example, [26, p. 71]; see also [8, Section 2.3] for more general SGclasses of symbols and operators on $\mathbb{R}^{d}$ as elements of the Weyl calculus).

\footnotetext{
${ }^{2}$ The formula involving integrals only holds true for $a \in \mathcal{S}\left(\mathbb{R}^{2 d}\right)$, but the quantization can be extended to any $a \in \mathcal{S}^{\prime}\left(\mathbb{R}^{2 d}\right)$, using the Fourier transform, the pullback by linear transformations, and the Schwartz kernel theorem.
} 
We list below some basic properties of SG-symbols and operators. (We refer to, for example, [4] and [30, Section 3.1] for an overview of the SGcalculus.) Some more information is provided in "Appendix", for the convenience of the reader.

1. OpSG $\left(\mathbb{R}^{d}\right)=\bigcup_{\left(m_{\psi}, m_{e}\right) \in \mathbb{R}^{2}} \mathrm{OpSG}^{m_{\psi}, m_{e}}\left(\mathbb{R}^{d}\right)$ is a graded *-algebra; its ele-

ments are linear continuous operators from $\mathcal{S}\left(\mathbb{R}^{d}\right)$ to itself, extendable to linear continuous operators from $\mathcal{S}^{\prime}\left(\mathbb{R}^{d}\right)$ to itself;

2. the differential operators of the form

$$
\sum_{|\alpha| \leq m_{e},|\beta| \leq m_{\psi}} a_{\alpha, \beta} x^{\alpha} D^{\beta}, \quad m_{e}, m_{\psi} \in \mathbb{N}_{0},
$$

are SG operators of order $\left(m_{\psi}, m_{e}\right)$;

3. if $A \in \mathrm{OpSG}^{0,0}\left(\mathbb{R}^{d}\right)$, then $A$ extends to a bounded linear operator

$$
A: L^{2}\left(\mathbb{R}^{d}\right) \rightarrow L^{2}\left(\mathbb{R}^{d}\right)
$$

4. there is an associated scale of SG-Sobolev spaces (also known as SobolevKato spaces), defined by

$$
H^{s_{\psi}, s_{e}}\left(\mathbb{R}^{d}\right)=\left\{u \in \mathcal{S}^{\prime}\left(\mathbb{R}^{d}\right):\left\|\langle\cdot\rangle^{s_{e}}\langle D\rangle^{s_{\psi}} u\right\|_{L^{2}\left(\mathbb{R}^{d}\right)}<\infty\right\},
$$

and for all $m_{\psi}, m_{e}, s_{\psi}, s_{e} \in \mathbb{R}$ the operator $A \in \mathrm{OpSG} m_{\psi}^{m_{e}}\left(\mathbb{R}^{d}\right)$ extends to a bounded linear operator

$$
A: H^{s_{\psi}, s_{e}}\left(\mathbb{R}^{d}\right) \rightarrow H^{s_{\psi}-m_{\psi}, s_{e}-m_{e}}\left(\mathbb{R}^{d}\right) ;
$$

5. the inclusions $H^{s_{\psi}, s_{e}}\left(\mathbb{R}^{d}\right) \subset H^{r_{\psi}, r_{e}}\left(\mathbb{R}^{d}\right), s_{\psi} \geq r_{\psi}, s_{e} \geq r_{e}$, are continuous, compact when the order components inequalities are both strict; moreover, the scale of the SG-Sobolev spaces is global, in the sense that

$$
\bigcup_{s_{\psi}, s_{e}} H^{s_{\psi}, s_{e}}\left(\mathbb{R}^{d}\right)=\mathcal{S}^{\prime}\left(\mathbb{R}^{d}\right), \quad \bigcap_{s_{\psi}, s_{e}} H^{s_{\psi}, s_{e}}\left(\mathbb{R}^{d}\right)=\mathcal{S}\left(\mathbb{R}^{d}\right) ;
$$

6. an operator $A=\mathrm{Op}^{w}(a) \in \mathrm{OpSG}^{m_{\psi}, m_{e}}\left(\mathbb{R}^{d}\right)$ is elliptic if its symbol $a$ is invertible for $|x|+|\xi| \geq R>0$, and $\chi(|x|+|\xi|)[a(x, \xi)]^{-1}$ is a symbol in $\mathrm{SG}^{-m_{\psi},-m_{e}}\left(\mathbb{R}^{2 d}\right)$, where $\chi \in \mathcal{C}^{\infty}(\mathbb{R})$ with $\chi(t)=1$ for $t>2 R$ and $\chi(t)=0$ for $t<R$;

7. if $A \in \mathrm{Op} \mathrm{SG}^{m_{\psi}, m_{e}}\left(\mathbb{R}^{d}\right)$ is an elliptic operator, then there is a parametrix $B \in \mathrm{OpSG} \mathrm{SG}^{-m_{\psi},-m_{e}}\left(\mathbb{R}^{d}\right)$ such that

$$
A B-\mathrm{I} \in \mathrm{OpSG}-\infty,-\infty\left(\mathbb{R}^{d}\right), \quad B A-\mathrm{I} \in \mathrm{OpSG}^{-\infty,-\infty}\left(\mathbb{R}^{d}\right) .
$$

\subsection{SG-Classical Symbols}

We first introduce two classes of SG-symbols which are homogeneous in the large with respect either to the variable or the covariable. For any $\rho>0$, $x_{0} \in \mathbb{R}^{d}$, we let $B_{\rho}\left(x_{0}\right)=\left\{x \in \mathbb{R}^{d}:\left|x-x_{0}\right|<\rho\right\}$ and we fix a cut-off function $\omega \in \mathcal{C}_{c}^{\infty}\left(\mathbb{R}^{d}\right)$ with $\omega \equiv 1$ on the ball $B_{\frac{1}{2}}(0)$. For proofs, we refer to $[30$, Section 3.2]. 
1. A symbol $a=a(x, \xi)$ belongs to the class $\mathrm{SG}_{\mathrm{cl}(\xi)}^{m_{\psi}, m_{e}}\left(\mathbb{R}^{2 d}\right)$ if there exist functions $a_{m_{\psi}-i, \cdot}(x, \xi), i=0,1, \ldots$, homogeneous of degree $m_{\psi}-i$ with respect to the variable $\xi$, smooth with respect to the variable $x$, such that,

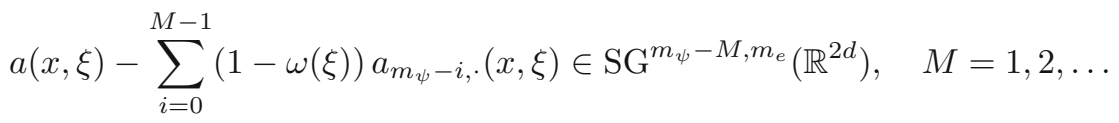

2. A symbol $a$ belongs to the class $\mathrm{SG}_{\mathrm{cl}(x)}^{m_{\psi}, m_{e}}\left(\mathbb{R}^{2 d}\right)$ if $a \circ R \in \mathrm{SG}_{\mathrm{cl}(\xi)}^{m_{e}, m_{\psi}}\left(\mathbb{R}^{2 d}\right)$, where $R(x, \xi)=(\xi, x)$. This means that $a(x, \xi)$ has an asymptotic expansion into homogeneous terms in $x$.

Definition 9. A symbol $a$ is called SG-classical, and we write $a \in \operatorname{SG}_{\operatorname{cl}(x, \xi)}^{m_{\psi}, m_{e}}\left(\mathbb{R}^{2 d}\right)$ $=\mathrm{SG}_{\mathrm{cl}}^{m_{\psi}, m_{e}}\left(\mathbb{R}^{2 d}\right)$, if the following two conditions hold true:

(i) there exist functions $a_{m_{\psi}-j, \cdot}(x, \xi)$, homogeneous of degree $m_{\psi}-j$ with respect to $\xi$ and smooth in $x$, such that $(1-\omega(\xi)) a_{m_{\psi}-j, \cdot}(x, \xi) \in$ $\mathrm{SG}_{\mathrm{cl}(x)}^{m_{\psi}-j, m_{e}}$

$\left(\mathbb{R}^{2 d}\right)$ and

$$
a(x, \xi)-\sum_{j=0}^{M-1}(1-\omega(\xi)) a_{m_{\psi}-j, \cdot}(x, \xi) \in \mathrm{SG}_{\mathrm{cl}(x)}^{m_{\psi}-M, m_{e}}\left(\mathbb{R}^{2 d}\right), \quad M=1,2, \ldots ;
$$

(ii) there exist functions $a_{\cdot, m_{e}-k}(x, \xi)$, homogeneous of degree $m_{e}-k$ with respect to the $x$ and smooth in $\xi$, such that $(1-\omega(x)) a_{\cdot, m_{e}-k}(x, \xi) \in$ $\mathrm{SG}_{\mathrm{cl}(\xi)}^{m_{\psi}, m_{e}-k}\left(\mathbb{R}^{2 d}\right)$ and

$$
a(x, \xi)-\sum_{k=0}^{M-1}(1-\omega(x)) a_{\cdot, m_{e}-k}(x, \xi) \in \mathrm{SG}_{\mathrm{cl}(\xi)}^{m_{\psi}, m_{e}-M}\left(\mathbb{R}^{2 d}\right), \quad M=1,2, \ldots
$$

Note that the definition of SG-classical symbol implies a condition of compatibility for the terms of the expansions with respect to $x$ and $\xi$. In fact, defining $\sigma_{m_{\psi}-j}^{\psi}$ and $\sigma_{m_{e}-i}^{e}$ on $\mathrm{SG}_{\mathrm{cl}(\xi)}^{m_{\psi}, m_{e}}$ and $\mathrm{SG}_{\mathrm{cl}(x)}^{m_{\psi}, m_{e}}$, respectively, as

$$
\begin{aligned}
& \sigma_{m_{\psi}-j}^{\psi}(a)(x, \xi)=a_{m_{\psi}-j, \cdot}(x, \xi), \quad j=0,1, \ldots, \\
& \sigma_{m_{e}-k}^{e}(a)(x, \xi)=a_{\cdot, m_{e}-k}(x, \xi), \quad k=0,1, \ldots,
\end{aligned}
$$

it possible to prove that (cf. $[30,(3.2 .7)]$ )

$$
a_{m_{\psi}-j, m_{e}-k}=\sigma_{m_{\psi}-j, m_{e}-k}^{\psi e}(a)=\sigma_{m_{\psi}-j}^{\psi}\left(\sigma_{m_{e}-k}^{e}(a)\right)=\sigma_{m_{e}-k}^{e}\left(\sigma_{m_{\psi}-j}^{\psi}(a)\right)
$$

for all $j, k \in \mathbb{N}_{0}$.

Moreover, the composition of two SG-classical operators is still classical. For $A=\mathrm{Op} a \in \mathrm{OpSG}_{\mathrm{cl}}^{m_{\psi}, m_{e}}\left(\mathbb{R}^{d}\right)$, the triple

$$
\sigma(A)=\left(\sigma^{\psi}(A), \sigma^{e}(A), \sigma^{\psi e}(A)\right)=\left(a_{\psi}, a_{e}, a_{\psi e}\right) .
$$


where

$$
\begin{gathered}
\sigma^{\psi}(A)(x, \xi)=a_{\psi}(x, \xi)=a_{m_{\psi}, \cdot}\left(x, \frac{\xi}{|\xi|}\right), \\
\sigma^{e}(A)(x, \xi)=a_{e}(x, \xi)=a_{\cdot, m_{e}}\left(\frac{x}{|x|}, \xi\right), \\
\sigma^{\psi, e}(A)(x, \xi)=a_{\psi e}(x, \xi)=a_{m_{\psi}, m_{e}}\left(\frac{x}{|x|}, \frac{\xi}{|\xi|}\right),
\end{gathered}
$$

is called the principal symbol of $A$. This definition keeps the usual multiplicative behaviour; that is, for any $A \in \mathrm{OpSG}_{\mathrm{cl}}^{m_{\psi}, m_{e}}\left(\mathbb{R}^{d}\right), B \in \mathrm{OpSG}_{\mathrm{cl}}^{r_{\psi}, r_{e}}\left(\mathbb{R}^{d}\right)$, $\left(m_{\psi}, m_{e}\right),\left(r_{\psi}, r_{e}\right) \in \mathbb{R}^{2}$, the principal symbol of $A B$ is given by

$$
\sigma(A B)=\sigma(A) \cdot \sigma(B)
$$

where the product is taken componentwise. Proposition 10 allows to express the ellipticity of SG-classical operators in terms of their principal symbol.

Fixing a cut-off function $\omega \in \mathcal{C}_{c}^{\infty}\left(\mathbb{R}^{d}\right)$ as above, we define the principal part of $a$ to be

$$
a_{p}(x, \xi)=(1-\omega(\xi)) a_{\psi}(x, \xi)+(1-\omega(x))\left(a_{e}(x, \xi)-(1-\omega(\xi)) a_{\psi e}(x, \xi)\right) .
$$

\subsection{SG-Wavefront Sets}

We denote by $\mathcal{W}$ the disjoint union

$$
\mathcal{W}=\mathcal{W}^{\psi} \sqcup \mathcal{W}^{e} \sqcup \mathcal{W}^{\psi e}=\left(\mathbb{R}^{d} \times \mathbb{S}^{d-1}\right) \sqcup\left(\mathbb{S}^{d-1} \times \mathbb{R}^{d}\right) \sqcup\left(\mathbb{S}^{d-1} \times \mathbb{S}^{d-1}\right),
$$

which may be viewed as the boundary of the (double) radial compactification of the phase space $T^{*} \mathbb{R}^{d} \simeq \mathbb{R}^{d} \times \mathbb{R}^{d}$ (see, for example, [4] and "Appendix"). Therefore, it is natural to define smooth functions on $\mathcal{W}$ as follows:

$$
\begin{aligned}
& \mathcal{C}^{\infty}(\mathcal{W})=\left\{\left(f_{\psi}, f_{e}, f_{\psi e}\right) \in \mathcal{C}^{\infty}\left(\mathcal{W}^{\psi}\right) \times \mathcal{C}^{\infty}\left(\mathcal{W}^{e}\right) \times \mathcal{C}^{\infty}\left(\mathcal{W}^{\psi e}\right):\right. \\
& \left.\quad \lim _{\lambda \rightarrow \infty} f_{\psi}(\lambda x, \xi)=\lim _{\lambda \rightarrow \infty} f_{e}(x, \lambda \xi)=f_{\psi e}(x, \xi) \text { for all }(x, \xi) \in \mathbb{S}^{d-1} \times \mathbb{S}^{d-1}\right\} .
\end{aligned}
$$

By restriction, the principal symbol can be defined as a map $\sigma: \mathrm{SG}_{\mathrm{cl}}^{m_{\psi}, m_{e}}$ $\left(\mathbb{R}^{2 d}\right) \ni a \mapsto \sigma(a) \in \mathcal{C}^{\infty}(\mathcal{W})$.

Proposition 10. An operator $A \in \mathrm{OpSG}_{\mathrm{cl}}^{m_{\psi}, m_{e}}\left(\mathbb{R}^{d}\right)$ is elliptic if and only if $\sigma(A)(x, \xi) \neq 0$ for all $(x, \xi) \in \mathcal{W}$.

For $A \in \mathrm{OpSG}_{\mathrm{cl}}^{m_{\psi}, m_{e}}\left(\mathbb{R}^{d}\right)$ we define the following sets (see $\left.[9,27]\right)$ :

1. the elliptic set

$$
\operatorname{ell}_{\mathrm{SG}}(A)=\{(x, \xi) \in \mathcal{W}: \sigma(A)(x, \xi) \neq 0\},
$$

2. the characteristic set

$$
\Sigma_{\mathrm{SG}}(A)=\mathcal{W} \backslash \operatorname{ell}_{\mathrm{SG}}(A)
$$


3. the operator $\mathrm{SG}$-wavefront set $\mathrm{WF}_{\mathrm{SG}}^{\prime}(A) \subset \mathcal{W}$, via its complement: $(x, \xi) \notin \mathrm{WF}_{\mathrm{SG}}^{\prime}(A)$ if there exists $B \in \mathrm{OpSG}_{\mathrm{cl}}^{0,0}\left(\mathbb{R}^{d}\right)$ such that $(x, \xi) \in$ ell $_{\mathrm{SG}}(B)$ and $A B \in \mathrm{Op} \mathrm{SG}-\infty,-\infty\left(\mathbb{R}^{d}\right)$. More concisely,

$$
\mathrm{WF}_{\mathrm{SG}}^{\prime}(A)=\bigcap_{\substack{B \in \mathrm{Op} \mathrm{SG}_{\mathrm{cl}}^{0,0} \\ A B \in \mathrm{Op} \mathrm{SG}}} \Sigma_{\mathrm{SG}}(B) .
$$

The SG-wavefront set of a distribution $u \in \mathcal{S}^{\prime}\left(\mathbb{R}^{d}\right)$ is defined as

$$
\mathrm{WF}_{\mathrm{SG}}(u)=\bigcap_{\substack{A \in \mathrm{Op} \mathrm{SG}_{\mathrm{cl}}^{0,0} \\ A u \in \mathcal{S}\left(\mathbb{R}^{d}\right)}} \Sigma_{\mathrm{SG}}(A),
$$

see $[4,9,27]$. Following the concept of wavefront space by Cordes (see [4, Sect. $2.3]$ ) and the approach in [9], we will decompose the SG-wavefront set of $u \in$ $\mathcal{S}^{\prime}\left(\mathbb{R}^{d}\right)$ into its components in $\mathcal{W}$, namely,

$\mathrm{WF}_{\mathrm{SG}}(u)=\left(\mathrm{WF}_{\mathrm{SG}}^{\psi}(u), \mathrm{WF}_{\mathrm{SG}}^{e}(u), \mathrm{WF}_{\mathrm{SG}}^{\psi e}(u)\right), \quad \mathrm{WF}_{\mathrm{SG}}^{\bullet}(u) \subset \mathcal{W}^{\bullet}, \bullet \in\{\psi, e, \psi e\}$.

Then, we have that

$$
\mathrm{WF}_{\mathrm{SG}}^{\psi}(u)=\mathrm{WF}_{\mathrm{cl}}(u),
$$

where $\mathrm{WF}_{\mathrm{cl}}(u)$ is the classical Hörmander's wavefront set.

The SG-wavefront set is well behaved with respect to the Fourier transform (see, for example, [9, Lemma 2.4]):

$$
(x, \xi) \in \mathrm{WF}_{\mathrm{SG}}(u) \Longleftrightarrow(\xi,-x) \in \mathrm{WF}_{\mathrm{SG}}(\widehat{u}) .
$$

\subsection{Complex Powers}

As in the case of closed manifolds, it is possible to define complex powers of SGpseudodifferential operators. We will only review the crucial properties of complex powers for a positive elliptic self-adjoint operator $A \in \mathrm{OpSG}_{\mathrm{cl}}^{m_{\psi}, m_{e}}\left(\mathbb{R}^{d}\right)$, $m_{\psi}, m_{e}>0$. For the definition and proofs of the following properties, we refer to [3, Proposition 2.8, Theorems 3.1 and 4.2] (cf. also [23,35]).

(i) $A^{z} A^{s}=A^{z+s}$ for all $z, s \in \mathbb{C}$.

(ii) $A^{k}=\underbrace{A \circ \ldots \circ A}_{k \text { times }}$ for $k \in \mathbb{N}_{0}$.

(iii) If $A \in \mathrm{OpSG}_{\mathrm{cl}}^{m_{\psi}, m_{e}}\left(\mathbb{R}^{d}\right)$, then $A^{z} \in \mathrm{OpSG}_{\mathrm{cl}}^{m_{\psi} \operatorname{Re} z, m_{e} \operatorname{Re} z}\left(\mathbb{R}^{d}\right)$.

(iv) If $A$ is a classical SG-operator, then $A^{z}$ is classical and its principal symbol is given by

$$
\sigma\left(A^{z}\right)=\sigma(A)^{z}
$$

(v) For $\operatorname{Re} z<-d \cdot \min \left\{1 / m_{e}, 1 / m_{\psi}\right\}, A^{z}$ is trace class.

For any $A=\mathrm{Op}(a) \in \mathrm{Op} \mathrm{SG} \mathrm{Sl}_{\mathrm{cl}}^{m_{\psi}, m_{e}}\left(\mathbb{R}^{d}\right)$ as above, the full symbol ${ }^{3}$ of $A^{z}$ will be denoted by

$$
a(z) \in \mathrm{OpSG}_{\mathrm{cl}}^{m_{\psi} \operatorname{Re} z, m_{e} \operatorname{Re} z}\left(\mathbb{R}^{d}\right) .
$$

\footnotetext{
${ }^{3}$ For the definition of the zeta function, it does not matter which quantization we choose.
} 
Let $s \in \mathbb{C}$ with $\operatorname{Re}(s)>\max \left\{d / m_{e}, d / m_{\psi}\right\}$. Using the property (v), it is possible to define $\zeta(s)$ by

$$
\zeta(s)=\operatorname{Tr} A^{-s}=\int K_{A^{-s}}(x, x) \mathrm{d} x=(2 \pi)^{-d} \iint a(x, \xi ;-s) \mathrm{d} x \mathrm{~d} \xi,
$$

where $K_{A^{z}}$ is the Schwartz kernel of $A^{z}$. We note that the $\zeta$-function may be written as

$$
\zeta(s)=\sum_{j=1}^{\infty} \lambda_{j}^{-s}
$$

with $\left(\lambda_{j}\right)_{j \in \mathbb{N}}$ the sequence of eigenvalues of $A$.

Theorem 11. (Battisti-Coriasco [3]) The function $\zeta(s)$ is holomorphic for $\operatorname{Re}(s)>d \cdot \max \left\{1 / m_{\psi}, 1 / m_{e}\right\}$. Moreover, it can be extended as a meromorphic function with possible poles at the points

$$
s_{j}^{1}=\frac{d-j}{m_{\psi}}, j=0,1, \ldots, \quad s_{k}^{2}=\frac{d-k}{m_{e}}, k=0,1, \ldots
$$

Such poles can be of order two if and only if there exist integers $j, k$ such that

$$
s_{j}^{1}=\frac{d-j}{m_{\psi}}=\frac{d-k}{m_{e}}=s_{k}^{2} .
$$

\subsection{Parametrix of SG-Hyperbolic Cauchy Problems}

Let $P \in \mathrm{OpSG}_{\mathrm{cl}}^{1,1}\left(\mathbb{R}^{d}\right)$ be a self-adjoint positive elliptic operator. By the construction from [11, Theorem 1.2] (cf. also [5,6,9]), it is possible to calculate a suitable parametrix for the Cauchy problem associated with the wave equation, namely

$$
\left\{\begin{aligned}
\left(i \partial_{t}-P\right) u(t, x) & =0 \\
u(0, x) & =u_{0}(x) .
\end{aligned}\right.
$$

The solution operator of (10) exists by the spectral theorem and is denoted by $U(t)=\mathrm{e}^{-i t P}=\left[\mathcal{F}_{\lambda \rightarrow t}(d E)\right](t)$, where $d E$ is the spectral measure of $P$. There exists a short-time parametrix $\widetilde{U}(t)$, which is given by a regular family of SG-Fourier integral operators of type I (cf. Section A.2 in "Appendix"), defined through the integral kernel

$$
K_{\widetilde{U}(t)}(x, y)=(2 \pi)^{-d} \int \mathrm{e}^{i(\phi(t, x, \xi)-y \xi)} \tilde{a}(t, x, \xi) \mathrm{d} \xi,
$$

where $\tilde{a} \in \mathcal{C}^{\infty}\left((-\epsilon, \epsilon), \mathrm{SG}_{\mathrm{cl}}^{0,0}\right)$ with $\tilde{a}(0)-1 \in \mathrm{SG}^{-\infty,-\infty}$ and $\phi \in \mathcal{C}^{\infty}((-\epsilon, \epsilon)$, $\left.\mathrm{SG}_{\mathrm{cl}}^{1,1}\right)$.

The parametrix $\widetilde{U}(t)$ solves the wave equation (10) in the sense that $\tilde{u}(t, x)=\left[\widetilde{U}(t) u_{0}\right](x)$ satisfies

$$
\left\{\begin{aligned}
\left(i \partial_{t}-P\right) \tilde{u}(t) & \in \mathcal{C}^{\infty}\left((-\epsilon, \epsilon), \mathcal{S}\left(\mathbb{R}^{d}\right)\right) \\
\tilde{u}(0)-u_{0} & \in \mathcal{S}\left(\mathbb{R}^{d}\right) .
\end{aligned}\right.
$$


By a Duhamel argument, $U(t)-\widetilde{U}(t) \in \mathcal{C}^{\infty}\left((-\epsilon, \epsilon), \mathcal{L}\left(\mathcal{S}^{\prime}\left(\mathbb{R}^{d}\right), \mathcal{S}\left(\mathbb{R}^{d}\right)\right)\right)$, (cf. [10, Theorem 16], [14, p. 284]). Since the error term is regularizing, we obtain that

$$
K_{U(t)}(x, y)=(2 \pi)^{-d} \int \mathrm{e}^{i(\phi(t, x, \xi)-y \xi)} a(t, x, \xi) \mathrm{d} \xi,
$$

for $a \in \mathcal{C}^{\infty}\left((-\epsilon, \epsilon), \mathrm{SG}_{\mathrm{cl}}^{0,0}\right)$ with $a(0)=1$ (cf. [7, Lemma 4.14]).

Let $p$ be the principal part of the full Weyl-quantized symbol of $P$. The phase function $\phi$ satisfies the eikonal equation

$$
\left\{\begin{aligned}
\partial_{t} \phi(t, x, \xi)+p\left(x, \phi_{x}^{\prime}(t, x, \xi)\right) & =0 \\
\phi(0, x, \xi) & =x \xi .
\end{aligned}\right.
$$

This implies that we have a Taylor expansion in $t$ of the form

$$
\phi(t, x, \xi)=x \xi-t p(x, \xi)+t^{2} \mathcal{C}^{\infty}\left(\mathbb{R}_{t}, \mathrm{SG}_{\mathrm{cl}}^{1,1}\right)
$$

for $t$ small enough.

For any $f \in \mathcal{C}^{\infty}\left(\mathbb{R}^{2 d}\right)$, we define the Hamiltonian vector field by

$$
\mathrm{X}_{f}=\left\langle\partial_{x} f, \partial_{\xi}\right\rangle-\left\langle\partial_{\xi} f, \partial_{x}\right\rangle
$$

and we denote its flow by $t \mapsto \exp \left(t \mathrm{X}_{f}\right)$. For $P \in \mathrm{OpSG}_{\mathrm{cl}}^{1,1}\left(\mathbb{R}^{d}\right)$, we will collectively denote by $\mathrm{X}_{\sigma(P)}$ the Hamiltonian vector fields on $\mathcal{W}^{\bullet}$ generated by $\sigma^{\bullet}(P), \bullet \in\{\psi, e, \psi e\}$, and by $t \mapsto \exp \left(t \mathrm{X}_{\sigma(P)}\right)$ the three corresponding flows.

By the group property, $U(t+s)=U(t) U(s)$, we can extend propagation of singularities results for small times to $t \in \mathbb{R}$. In [9], the propagation of the SGwavefront set under the action of SG-classical operators and operator families like $U(t)$ has been studied. In particular, the following theorem was proved there, by means of SG-Fourier integral operators (see also [8] and the principaltype propagation result for the scattering wavefront set [27, Proposition 7]).

Theorem 12. Let $u_{0} \in \mathcal{S}^{\prime}\left(\mathbb{R}^{d}\right)$ and $U(t)=e^{-i t P}$. Then,

$$
\mathrm{WF}_{\mathrm{SG}}^{\bullet}\left(U(t) u_{0}\right) \subseteq \Phi^{\bullet}(t)\left(\mathrm{WF}_{\mathrm{SG}}^{\bullet}\left(u_{0}\right)\right),
$$

where $\Phi^{\bullet}$ is the smooth family of canonical transformations on $\mathcal{W}^{\bullet}$ generated by $\sigma^{\bullet}(\phi)$ with $\bullet \in\{\psi, e, \psi e\}$.

Remark 13. In view of (15), Theorem 12 can also be stated in the following way: For any $u_{0} \in \mathcal{S}^{\prime}\left(\mathbb{R}^{d}\right)$ and $t \in(-\epsilon / 2, \epsilon / 2), \mathrm{WF}_{\mathrm{SG}}^{\bullet}\left(U(t) u_{0}\right) \subset \exp \left(t \mathrm{X}_{\sigma} \bullet(p)\right)$ $\left(\mathrm{WF}_{\mathrm{SG}}^{\bullet}\left(u_{0}\right)\right)$, where $\bullet \in\{\psi, e, \psi e\}$, and $\mathrm{X}_{f}$ is the Hamiltonian vector field generated by $f$. In the sequel, we will express this fact in the compact form

$$
\mathrm{WF}_{\mathrm{SG}}\left(U(t) u_{0}\right) \subset \exp \left(t \mathrm{X}_{\sigma(p)}\right)\left(\mathrm{WF}_{\mathrm{SG}}\left(u_{0}\right)\right), \quad u_{0} \in \mathcal{S}^{\prime}\left(\mathbb{R}^{d}\right), t \in \mathbb{R} .
$$

\subsection{SG-Operators on Manifolds}

In the 80s, Schrohe [34] introduced the class of SG-manifolds, whose elements $\operatorname{admit} \mathcal{C}^{\infty}$ structures associated with finite atlases, where the changes of coordinates satisfy suitable estimates of SG-type. This class includes non-compact manifolds, namely, for instance, the Euclidean space $\mathbb{R}^{d}$, the infinite-holed torus (see, for example, [36, Page 25]), and the manifolds with ends (see, for example, 
[36, Page 27]), including those considered in [3,22] (see Section A.4 in "Appendix"). In particular, Schrohe showed that the concepts of Schwartz functions and distributions, as well as of weighted Sobolev space, have an invariant meaning on SG-manifolds, and the same holds true for SG-operators. In fact, the corresponding symbol and operator classes are preserved by the admissible changes of coordinates (cf. also [5, Section 4.4], for an alternative proof of this property). Maniccia and Panarese [22] considered a class of manifolds with ends and showed that a type of SG-classical operators can be defined there, with the principal symbol triple having an invariant meaning. In [3], this was employed, with reference to SG-classical operators locally described by the symbols recalled in Sect. 2.1, to study the $\zeta$-function of SG-operators on manifolds with ends satisfying suitable ellipticity properties, as well as to extend to such environment the concepts of Wodzicki residue and of the trace operators introduced by Nicola [31]. The latter appear in the statements of Theorems 1 and 2. The classical SG-operators have also been employed by Schulze (see, for example, [37]), with the terminology symbols with exit behaviour, in some steps of the construction of pseudodifferential calculi on singular manifolds.

Melrose $[26,27]$ introduced the so-called scattering calculus on asymptotically Euclidean manifolds. As it can be seen in the quoted references (see, for example, [26, Sect. 6.3]), up to a different choice of compactification with respect to the one we employ (cf. Section A.1 in "Appendix"), and an opposite sign for the $e$-order of the symbols (that is, the order with respect to the $x$ variable), the operators belonging to the scattering calculus are locally represented by SG pseudodifferential operators. In particular, the principal symbol of the classical operators has an invariant meaning, and the same holds true for the wavefront set (about the latter, see also the comparison in [9, Section 6] between the scattering wavefront set and the SG-wavefront set on $\mathbb{R}^{d}$ recalled in Sect. 2.2).

In view of the above observations about the invariance property of the SGcalculus on asymptotically Euclidean manifolds, in the sequel we will mostly work and prove our results for the locally defined operators, that is, on $\mathbb{R}^{d}$, with the global results following by a partition of unity and local coordinates argument.

\section{Wave Trace}

We fix a positive elliptic operator $P \in \mathrm{OpSG}_{\mathrm{cl}}^{1,1}\left(\mathbb{R}^{d}\right)$ with $\psi e$-principal symbol $p_{\psi e}=\sigma^{\psi e}(P)$. By the compactness of the embedding of SG-Sobolev spaces, we have that the resolvent $(\lambda-P)^{-1}$ is compact for $\lambda>0$, and hence, there exists an orthonormal basis $\left\{\psi_{j}\right\}$ of $L^{2}$ consisting of eigenfunctions of $P$ with eigenvalues $\lambda_{j}$ with the property that

$$
0<\lambda_{1} \leq \lambda_{2} \leq \cdots \rightarrow+\infty
$$


Therefore, the spectral measure is given by $d E(\lambda)=\sum_{j=1}^{\infty} \delta_{\lambda_{j}}(\lambda)\left\langle\cdot, \psi_{j}\right\rangle \psi_{j}$, where $\delta_{\mu}$ is the delta distribution centred at $\mu$, and we have that

$$
N(\lambda)=\operatorname{Tr} \int_{0}^{\lambda} d E(\lambda)=\operatorname{Tr} E_{\lambda} .
$$

The wave trace $w(t)$ is (formally) defined as

$$
w(t)=\operatorname{Tr} U(t)=\sum_{j=1}^{\infty} \mathrm{e}^{-i t \lambda_{j}} .
$$

As usual, $w(t)$ is well defined as a distribution by means of integration by parts and the fact that $P^{-N}$ is trace class for $N>d$ (cf. Schrohe [35, Theorem 2.4]). Theorem 12 directly implies Lemma 14.

Lemma 14. Choose $t_{0} \in \mathbb{R}$. Let $\Gamma \subset \mathcal{W}$ be an open subset and such that $\left[\exp \left(t \mathrm{X}_{\sigma(P)}\right)(\Gamma)\right] \cap \Gamma=\emptyset$, for all $t \in\left(t_{0}-\delta, t_{0}+\delta\right)$ and $\delta>0$ small. Then, for all $B \in \mathrm{Op} \mathrm{SG} \mathrm{Cl}_{\mathrm{cl}}^{0,0}\left(\mathbb{R}^{d}\right)$ with $\mathrm{WF}_{\mathrm{SG}}^{\prime}(B) \subset \Gamma$, and all $t \in\left(t_{0}-\delta, t_{0}+\delta\right)$, we have that $B U(t) B \in \mathcal{L}\left(\mathcal{S}^{\prime}\left(\mathbb{R}^{d}\right), \mathcal{S}\left(\mathbb{R}^{d}\right)\right)$.

We will show that the improvement of the Weyl law is only related to the corner component

$$
\left\{t \in \mathbb{R}: \exp \left(t \mathbf{X}_{\sigma_{\psi e}(P)}\right)(x, \xi)=(x, \xi) \text { for some }(x, \xi) \in \mathcal{W}^{\psi e}\right\} .
$$

The structure of the singularities of $w(t)$ is more involved. This comes from the fact that the boundary at infinity is not a manifold or equivalently the flow is not homogeneous. In contrast to the case of a closed manifold, the distribution $w(t)$ will not be a conormal distribution near 0 , but it turns out that it is a log-polyhomogeneous distribution.

Let $\epsilon>0$ as in Sect. 2.4 and choose a function $\chi \in \mathcal{S}(\mathbb{R})$ with supp $\hat{\chi} \subset$ $(-\epsilon, \epsilon)$ and $\hat{\chi}=1$ on $(-\epsilon / 2, \epsilon / 2)$.

Proposition 15. Let $B \in \mathrm{OpSG}_{\mathrm{cl}}^{0,0}$ and denote by $N_{B}(\lambda)=\operatorname{Tr}\left(E_{\lambda} B B^{*}\right)$ the microlocalized counting function. There exist coefficients $w_{j k} \in \mathbb{R}$ with $k \in \mathbb{N}_{0}$ and $j \in\{0,1\}$ independent of $\chi$ such that

$$
\left(N_{B} * \chi\right)(\lambda) \sim \sum_{k=0}^{\infty} \sum_{j=0,1} w_{j k} \lambda^{d-k}(\log \lambda)^{j}
$$

as $\lambda \rightarrow \infty$.

Remark 16. Note that $\left[\mathcal{F}\left(N_{B}^{\prime}\right)\right](t)=\operatorname{Tr}\left(U(t) B B^{*}\right)$.

Proof. From Sect. 2.4, through the calculus of SG FIOs (see [5] and Section A.2 in "Appendix"), we obtain that there is a parametrix $\widetilde{U}(t)$ for $U(t)$ and we have the local representation of the kernel

$$
K_{U(t) B B^{*}}(x, y)=(2 \pi)^{-d} \int \mathrm{e}^{i(\phi(t, x, \xi)-y \xi)} a(t, x, \xi) \mathrm{d} \xi
$$

for $t \in(-\epsilon, \epsilon)$. The amplitude satisfies $\sigma(a(0))=\sigma\left(B B^{*}\right)$. 
Set

$$
\mathcal{T}_{B}(t)=\hat{\chi}(t) \operatorname{Tr}\left(U(t) B B^{*}\right) .
$$

By the previous remark, we have that $\mathcal{T}_{B}(t)$ is the Fourier transform of $\left(N_{B}^{\prime} *\right.$ $\chi)(\lambda)$. We will now calculate the inverse Fourier transform of $\mathcal{T}_{B}$.

Using the Taylor expansion of the phase function, we have that

$$
\phi(t, x, \xi)=x \xi+t \psi(t, x, \xi),
$$

where $\psi$ is smooth in $t$. Formally, we can write the trace as

$$
\mathcal{T}_{B}(t)=(2 \pi)^{-d} \hat{\chi}(t) \int \mathrm{e}^{i t \psi(t, x, \xi)} a(t, x, \xi) \mathrm{d} x \mathrm{~d} \xi
$$

We proceed as in Hörmander [20, pp. 254-256], and we set

$$
\tilde{A}_{B}(t, \lambda)=(2 \pi)^{-d} \hat{\chi}(t) \int_{\{-\psi(t, x, \xi) \leq \lambda\}} a(t, x, \xi) \mathrm{d} x \mathrm{~d} \xi .
$$

Note that ellipticity implies that $\tilde{A}_{B}(t, \lambda)<\infty$.

Set $X=\mathbb{B}^{d}$ and ${ }^{\mathrm{sc}} \bar{T}^{*} X=\mathbb{B}^{d} \times \mathbb{B}^{d}$ with boundary defining functions $\rho_{X}$ and $\rho_{\Xi}$ as explained in Section A.1 of "Appendix", and let

$$
u(t, x, \xi)=(2 \pi)^{-d} \hat{\chi}(t) a(t, x, \xi) \mathrm{d} x \mathrm{~d} \xi .
$$

Under the compactification $\iota_{2}=\iota \times \iota: \mathbb{R}^{d} \times \mathbb{R}^{d} \rightarrow \mathbb{B}^{d} \times \mathbb{B}^{d}={ }^{\mathrm{sc}} \bar{T}^{*} X$, we have that

$$
\left(\iota_{2}\right)_{*} u \in \rho_{X}^{-d} \rho_{\Xi}^{-d} \mathcal{C}^{\infty}\left({ }^{\mathrm{sc}} \bar{T}^{*} X,{ }^{b} \Omega\right) .
$$

In the language of Melrose [25], $\left(\iota_{2}\right)_{*} u \in \mathcal{A}_{\mathrm{phg}}^{\mathcal{K}}\left({ }^{\mathrm{sc}} \bar{T}^{*} X,{ }^{b} \Omega\right)$ with index set $\mathcal{K}$ given by $K\left(\left\{\rho_{X}=0\right\}\right)=K\left(\left\{\rho_{\Xi}=0\right\}\right)=\left(-d+\mathbb{N}_{0}\right) \times\{0\}$.

It follows from (17) that

$$
\begin{aligned}
\partial_{\lambda} \tilde{A}_{B}(t, \lambda) & =\int_{-\psi=\lambda} u \\
& =\left\langle(-\psi)_{*} u, \delta_{\lambda}\right\rangle .
\end{aligned}
$$

The function $\tilde{\psi}(t)=\left(\iota \circ(-\psi) \circ \iota_{2}^{-1}\right)(t):{ }^{\mathrm{sc}} \bar{T}^{*} X \rightarrow \mathbb{B}^{1}=: \overline{\mathbb{R}}$ is a b-fibration with exponent matrix $(1,1)$ since the symbol $\psi$ is of order $(1,1)$. Hence, the Push-Forward Theorem (cf. Melrose [25] and Grieser and Gruber [17]) implies that

$$
(\iota \circ(-\psi))_{*} u \in \mathcal{A}_{\mathrm{phg}}^{\psi_{\#} \mathcal{K}}\left(\overline{\mathbb{R}},{ }^{b} \Omega\right),
$$

where $\psi_{\#} \mathcal{K}=-d+\mathbb{N}_{0} \times\{0,1\}$. Recall that the bundle ${ }^{b} \Omega$ is generated by $\rho^{-1} d \rho$ and $d y_{j}$ near the boundary $\{\rho=0\}$.

Pulling this distribution back to $\mathbb{R}$ and pairing with $\delta_{\lambda}$, we obtain the asymptotics

$$
\partial_{\lambda} \tilde{A}_{B}(t, \lambda) \sim \sum_{k=0}^{\infty} \sum_{j=0,1} a_{j k}(t) \lambda^{d-1-k}(\log \lambda)^{j}+O\left(\lambda^{-\infty}\right),
$$


where the coefficients $a_{j k}$ are smooth and compactly supported in $t$. Defining $A_{B}(\lambda)=\left.\mathrm{e}^{i D_{t} D_{\lambda}} \tilde{A}_{B}(t, \lambda)\right|_{t=0}$, we find

$$
\mathcal{T}_{B}(t)=\int_{\mathbb{R}} \mathrm{e}^{-i t \lambda} \partial_{\lambda} A_{B}(\lambda) \mathrm{d} \lambda .
$$

The above calculation implies that $\tilde{A}_{B}$ and $A_{B}$ have the asymptotics

$$
A_{B}(\lambda)=\sum_{k=0}^{\infty} \sum_{j=0,1} w_{j k} \lambda^{d-k}(\log \lambda)^{j}+O\left(\lambda^{-\infty}\right) .
$$

We conclude that

$$
\begin{aligned}
\left(N_{B} * \chi\right)(\lambda) & =\int_{-\infty}^{\lambda} \mathcal{F}_{t \rightarrow \lambda}^{-1}\left\{\mathcal{T}_{B}\right\}(\lambda) \mathrm{d} \lambda \\
& =A_{B}(\lambda) \\
& =\sum_{k=0}^{\infty} \sum_{j=0,1} w_{j k} \lambda^{d-k}(\log \lambda)^{j}+O\left(\lambda^{-\infty}\right) .
\end{aligned}
$$

We note that the coefficients are determined by derivatives of $\tilde{A}_{B}(t, \lambda)$ at $t=0$ and since $\hat{\chi}=1$ near $t=0$, the specific choice of $\chi$ does not change the coefficients.

\section{Relation with the Spectral $\zeta$-Function}

As in the case of pseudodifferential operators on closed manifolds (cf. Duistermaat and Guillemin [15, Corollary 2.2]), the wave trace at $t=0$ is related to the spectral $\zeta$-function. This relation extends to the SG setting.

Recall that for a positive self-adjoint elliptic operator $P \in \mathrm{OpSG}_{\mathrm{cl}}^{1,1}\left(\mathbb{R}^{d}\right)$, the function $\zeta(s)$ is defined for $\operatorname{Re} s>d$ by

$$
\zeta(s)=\operatorname{Tr} P^{-s} .
$$

In addition, we consider the microlocalized version of $\zeta(s)$, defined by

$$
\zeta_{B}(s)=\operatorname{Tr}\left(P^{-s} B B^{*}\right)=\sum_{j=1}^{\infty} \lambda_{j}^{-s}\left\|B^{*} \psi_{j}\right\|^{2}, \quad \operatorname{Re} s>d,
$$

for $B \in \mathrm{OpSG}_{\mathrm{cl}}^{0,0}$. Of course, $\zeta_{\mathrm{I}}(s)=\zeta(s)$.

By Theorem 11, $\zeta(s)$ admits a meromorphic continuation to $\mathbb{C}$ with poles of maximal order two at $d-k, k \in \mathbb{N}_{0}$. This result extends to $\zeta_{B}(s)$, and we characterize the Laurent coefficients in terms of the wave trace expansion at $t=0$.

Proposition 17. The function $\zeta_{B}(s)$ extends meromorphically to $\mathbb{C}$ and has at most poles of order two at the points $d-k, k \in \mathbb{N}_{0}$. We have the expansion

$$
\zeta_{B}(s)=\frac{A_{2, k}}{[s-(d-k)]^{2}}+\frac{A_{1, k}}{s-(d-k)}+f(s),
$$


where $f$ is holomorphic near $s=d-k$ and

$$
\begin{aligned}
& A_{2, k}=(d-k) w_{1 k}, \\
& A_{1, k}=w_{1 k}+(d-k) w_{0 k},
\end{aligned}
$$

where the $w_{j k}, k \in \mathbb{N}_{0}, j=0,1$, are the coefficients appearing in the asymptotic expansion (16) of $N_{B}(\lambda)$.

Proof. The meromorphic continuation and the possible location of the poles follow from similar arguments as in [3, Theorem 3.2] (see also the proof of Proposition 19). Hence, we only have to show that the poles are related to $N_{B}(\lambda)$.

Let $\epsilon \in\left(0, \lambda_{1}\right)$ be sufficiently small. Choose an excision function $\chi \in$ $\mathcal{C}^{\infty}(\mathbb{R})$ such that $\chi(\lambda)=0$ for $\lambda<\epsilon$ and $\chi(\lambda)=1$ for $\lambda \geq \lambda_{1}$. Set $\chi_{s}(\lambda)=$ $\chi(\lambda) \lambda^{-s}$. Then, using Remark 16,

$$
\zeta_{B}(s)=\left\langle N_{B}^{\prime}, \chi_{s}\right\rangle=\left\langle\operatorname{Tr}\left(U(t) B B^{*}\right), \mathcal{F}^{-1}\left(\chi_{s}\right)\right\rangle .
$$

Let $\rho \in \mathcal{S}(\mathbb{R})$ such that $\rho$ is positive, $\hat{\rho}(0)=1, \hat{\rho} \in \mathcal{C}_{c}^{\infty}(\mathbb{R})$, and $\rho$ is even. By an argument similar to the one in [15, Corollary 2.2], we have that

$$
\zeta_{B}(s)-\left\langle N_{B}^{\prime} * \rho, \chi_{s}\right\rangle=\left\langle(1-\hat{\rho}) \operatorname{Tr}\left(U(t) B B^{*}\right), \mathcal{F}^{-1}\left(\chi_{s}\right)\right\rangle
$$

is entire in $s$ and polynomially bounded for $\operatorname{Re} s>C$.

Now, we can insert the asymptotic expansion of $N_{B}^{\prime} * \rho$ to calculate the residues of $\zeta_{B}(s)$. Taking the derivative of (16), we see that the asymptotic expansion of $N_{B}^{\prime} * \rho$ is given by

$$
\left(N_{B}^{\prime} * \rho\right)(\lambda)=\sum_{k=0}^{N} \sum_{j=0,1} A_{j+1, k} \lambda^{d-k-1}(\log \lambda)^{j}+o\left(\lambda^{d-1-N}\right)
$$

for any $N \in \mathbb{N}_{0}$ and $A_{j, k}$ are given by (18).

Let $k \in \mathbb{N}_{0}$ be arbitrary. If $f \in \mathcal{C}^{\infty}(\mathbb{R})$ with $f(\lambda)=O\left(\lambda^{d-k-1} \log \lambda\right)$ as $\lambda \rightarrow \infty$, then $\int f(\lambda) \chi(\lambda) \lambda^{-s} \mathrm{~d} \lambda$ is bounded and holomorphic in $s$ for $\operatorname{Re} s>$ $d-k$. Let

$$
I(s)=\int \lambda^{d-k-1} \chi(\lambda) \lambda^{-s} \mathrm{~d} \lambda .
$$

By partial integration, we obtain

$$
I(s)=\frac{\psi(s)}{s-(d-k)} .
$$

where $\psi(s)=\int \lambda^{d-k-s} \chi^{\prime}(\lambda) \mathrm{d} \lambda$ is holomorphic and $\psi(d-k)=1$. Therefore, we have

$$
\int \lambda^{d-k-s-1}\left(A_{1, k}+A_{2, k} \log \lambda\right) \chi(\lambda) \mathrm{d} \lambda=-A_{2, k} I^{\prime}(s)+A_{1, k} I(s) .
$$

Hence, the integral near $s=d-k$ is given by

$$
\int \lambda^{d-k-s-1}\left(A_{1, k}+A_{2, k} \log \lambda\right) \chi(\lambda) \mathrm{d} \lambda=\frac{A_{2, k}}{[s-(d-k)]^{2}}+\frac{A_{1, k}}{s-(d-k)}+f(s),
$$


where $f$ is holomorphic in a neighbourhood of $s=d-k$. This shows that $A_{k, 2}$ and $A_{k, 1}$ as defined by (18) are the Laurent coefficients of $\zeta(s)$ at $s=d-k$.

The main advantage in employing the $\zeta$-function is that the coefficients are easier to calculate than for the wave trace.

Proposition 18. Let $B \in \mathrm{OpSG}_{\mathrm{cl}}^{0,0}$ with principal $\psi$ e-symbol $b_{\psi e}$. The function $\zeta_{B}(s)$ has a pole of order two at $s=d$ with leading Laurent coefficient

$$
(2 \pi)^{-d} \int_{\mathbb{S}^{d-1}} \int_{\mathbb{S}^{d-1}}\left[p_{\psi e}(\theta, \omega)\right]^{-d} \cdot b_{\psi e}(\theta, \omega) \mathrm{d} \theta \mathrm{d} \omega .
$$

Proof. This follows from the same arguments as in [3, Theorems 3.2 and 4.2] (cf. the proof of Proposition 19), with the modification that the full symbol is $a(z)=p(z) \# b$, where $p(z)$ denotes the full symbol of $P^{z}$. The principal $\psi e-$ symbol of $A(z)=P^{z} B$ is given by $a_{z, z}(x, \xi ; z)=\left[p_{\psi e}(x, \xi)\right]^{z} \cdot b_{\psi e}(x, \xi)$.

For the three-term asymptotics, we compute the third coefficient more explicitly.

Proposition 19. Let $p(s)=p(x, \xi ; s)$ be the full symbol of $P^{s}$. The leading Laurent coefficient of $\zeta(s)$ at $s=d-1$ is given by

$$
A_{2,1}=(2 \pi)^{-d} \int_{\mathbb{S}^{d-1}} \int_{\mathbb{S}^{d-1}} p_{-d,-d}(\theta, \omega ;-d+1) \mathrm{d} \theta \mathrm{d} \omega=\operatorname{TR}\left(P^{-(d-1)}\right) .
$$

Remark 20. The equality $A_{2,1}=\operatorname{TR}\left(P^{-(d-1)}\right)$ also holds on any manifold with cylindrical ends.

Proof. The second equality follows directly from Proposition 40 in "Appendix".

As in [3], we split the zeta function into four parts

$$
\zeta(s)=\sum_{j=1}^{4} \zeta_{j}(s),
$$

where, for $\operatorname{Re} s>d$,

$$
\zeta_{j}(s)=(2 \pi)^{-d} \int_{\Omega_{j}} p(x, \xi ;-s) d x d \xi
$$

and

$$
\begin{array}{ll}
\Omega_{1}=\{(x, \xi):|x| \leq 1,|\xi| \leq 1\}, & \Omega_{2}=\{(x, \xi):|x| \leq 1,|\xi|>1\} \\
\Omega_{3}=\{(x, \xi):|x|>1,|\xi| \leq 1\}, & \Omega_{4}=\{(x, \xi):|x|>1,|\xi|>1\} .
\end{array}
$$

Let us recall the main aspects of the proof of the properties of the four terms $\zeta_{j}(s), j=1, \ldots, 4$, shown in [3].

1. $\zeta_{1}(s)$ is holomorphic, since we integrate $p(-s)$, a holomorphic function in $s$ and smooth with respect to $(x, \xi)$, on a bounded set with respect to $(x, \xi)$. 
2. Let us first assume $\operatorname{Re} s>d$. Using the expansion of $p(-s)$ with $M \geq 1$ terms homogeneous with respect to $\xi$, switching to polar coordinates in $\xi$ and integrating the radial part, one can write

$$
\begin{aligned}
\zeta_{2}(s)= & (2 \pi)^{-d} \sum_{j=0}^{M-1} \frac{1}{s-(d-j)} \int_{|x| \leq 1} \int_{\mathbb{S}^{d-1}} p_{-s-j, \cdot}(x, \omega ;-s) \mathrm{d} \omega \mathrm{d} x \\
& +(2 \pi)^{-d} \iint_{\Omega_{2}} r_{-s-M, \cdot}(x, \xi ;-s) \mathrm{d} \xi \mathrm{d} x .
\end{aligned}
$$

Notice that the last integral is convergent and provides a holomorphic function in $s$. Arguing similarly to the case of operators on smooth, compact manifolds, $\zeta_{2}(s)$ turns out to be holomorphic for $\operatorname{Re}(s)>d$, extendable as a meromorphic function to the whole complex plane with, at most, simple poles at the points $s_{j}^{1}=d-j, j=0,1,2, \ldots$

3. Using now the expansion of $p(-s)$ with respect to $x$, exchanging the role of variable and covariable with respect to the previous point, again first assuming $\operatorname{Re} s>d$ and choosing $M \geq 1$, one can write

$$
\begin{aligned}
\zeta_{3}(s)= & (2 \pi)^{-d} \sum_{k=0}^{M-1} \frac{1}{s-(d-k)} \int_{\mathbb{S}^{d-1}} \int_{|\xi| \leq 1} p \cdot,-s-k(\theta, \xi ;-s) \mathrm{d} \xi \mathrm{d} \theta \\
& +(2 \pi)^{-d} \iint_{\Omega_{3}} t \cdot,-s-M(x, \xi ;-s) \mathrm{d} \xi \mathrm{d} x .
\end{aligned}
$$

Arguing as in point $2, \zeta_{3}(s)$ turns out to be holomorphic for $\operatorname{Re} s>d$, extendable as a meromorphic function to the whole complex plane with, at most, simple poles at the points $s_{k}^{2}=d-k, k=0,1,2, \ldots$

4. To treat the last term, both the expansions with respect to $x$ and with respect to $\xi$ are needed. We assume that $\operatorname{Re} s>d$ and choose $M \geq 1$. We argue as in point 2 to obtain

$$
\begin{aligned}
\zeta_{4}(s)= & (2 \pi)^{-d} \sum_{j=0}^{M-1} \frac{1}{s-(d-j)} \int_{|x| \geq 1} \int_{\mathbb{S}^{d-1}} p_{-s-j, \cdot}(x, \omega ;-s) \mathrm{d} \omega \mathrm{d} x \\
& +(2 \pi)^{-d} \iint_{\Omega_{4}} r_{-s-M, \cdot}(x, \xi ;-s) \mathrm{d} \xi \mathrm{d} x .
\end{aligned}
$$

Now, we introduce the expansion with respect to $x$, switching to polar coordinates and integrating the $x$-radial variable in the homogeneous terms, for both integrals

$$
\begin{aligned}
\int_{|x| \geq 1} \int_{\mathbb{S}^{d-1}} p_{-s-j, \cdot}(x, \omega ;-s) \mathrm{d} \omega \mathrm{d} x \\
=\sum_{k=0}^{M-1} \frac{1}{s-(d-k)} \int_{\mathbb{S}^{d-1}} \int_{\mathbb{S}^{d-1}} p_{-s-j,-s-k}(\theta, \omega ;-s) \mathrm{d} \theta \mathrm{d} \omega \\
\quad+\int_{|x| \geq 1} \int_{\mathbb{S}^{d-1}} t_{-s-j,-s-M}(x, \omega ;-s) \mathrm{d} x \mathrm{~d} \omega
\end{aligned}
$$


and

$$
\begin{aligned}
& \iint_{\Omega_{4}} r_{-s-M, \cdot}(x, \xi ;-s) \mathrm{d} \xi \mathrm{d} x \\
& =\sum_{k=0}^{M-1} \frac{1}{s-(d-k)} \int_{\mathbb{S}^{d-1}} \int_{|\xi| \geq 1} r_{-s-M,-s-k}(\theta, \xi ;-s) \mathrm{d} \xi \mathrm{d} \theta \\
& +\iint_{\Omega_{4}} r_{-s-M,-s-M}(x, \xi ;-s) \mathrm{d} x \mathrm{~d} \xi .
\end{aligned}
$$

We end up with

$$
\begin{aligned}
\zeta_{4}(s)= & \sum_{k=0}^{M-1} \sum_{j=0}^{M-1} \frac{1}{s-(d-j)} \frac{1}{s-(d-k)} I_{j}^{k}(s) \\
& +\sum_{j=0}^{M-1} \frac{1}{s-(d-j)} R_{j}^{M}(s)+\sum_{k=0}^{M-1} \frac{1}{s-(d-k)} R_{M}^{k}(s)+R_{M}^{M}(s),
\end{aligned}
$$

where

$$
I_{j}^{k}(s)=(2 \pi)^{-d} \int_{\mathbb{S}^{d-1}} \int_{\mathbb{S}^{d-1}} p_{-s-j,-s-k}\left(\theta^{\prime}, \theta ;-s\right) \mathrm{d} \theta \mathrm{d} \theta^{\prime},
$$

and $R_{M}^{j}, R_{k}^{M}, R_{M}^{M}$ are holomorphic in $s$ for $\operatorname{Re} s>M+d, j, k=0, \ldots, M-1$. It follows that $\zeta_{4}(s)$ is holomorphic for $\operatorname{Re}(s)>d$ and can be extended as a meromorphic function to the whole complex plane with, at most, poles at the points $s_{j}^{1}=d-j, s_{k}^{2}=d-k$ with $j, k \in \mathbb{N}_{0}$. Clearly, such poles can be of order two if and only if $j=k$ (cf. Theorem 11).

In view of the properties of $\zeta(s)$ recalled above, the limit

$$
\lim _{s \rightarrow d-1}[s-(d-1)]^{2} \zeta(s)=\lim _{s \rightarrow d-1}[s-(d-1)]^{2} \zeta_{4}(s)=I_{1}^{1}(d-1)
$$

proves the desired claim.

\section{Proof of the Main Theorems}

We choose a positive function $\rho \in \mathcal{S}(\mathbb{R})$ such that $\hat{\rho}(0)=1$, supp $\hat{\rho} \subset[-1,1]$, and $\rho$ is even. For $T>0$, we set $\rho_{T}(\lambda):=T \rho(T \lambda)$, which implies that $\hat{\rho_{T}}(t)=$ $\hat{\rho}(t / T)$. Let $\nu>0$ be arbitrary. Then, it is possible to prove the next Tauberian theorem by following the proof in [33, Appendix B].

Theorem 21. (Tauberian theorem) Let $N: \mathbb{R} \rightarrow \mathbb{R}$ such that $N$ is monotonically non-decreasing, $N(\lambda)=0$ for $\lambda \leq 0$, and is polynomially bounded as $\lambda \rightarrow+\infty$. If

$$
\left(\partial_{\lambda} N * \rho_{T}\right)(\lambda) \leq C_{1} \lambda^{\nu} \log \lambda, \quad \lambda \geq T^{-1}
$$

for $C_{1}>0$, then

$$
\left|N(\lambda)-\left(N * \rho_{T}\right)(\lambda)\right| \leq C C_{1} T^{-1} \lambda^{\nu} \log \lambda, \quad \lambda \geq T^{-1} .
$$


Proof of Theorem 1. The first part of Theorem 1 follows directly from the Tauberian theorem and Proposition 15, due to the identity

$$
\left[\mathcal{F}\left(N^{\prime}\right)\right](t)=\operatorname{Tr} \mathrm{e}^{-i t P}
$$

and using a partition of unity to locally represent $U(t)=\mathrm{e}^{-i t P}$ as a smooth family of SG-Fourier integral operators, as discussed in Sect. 2.4. To calculate the coefficients $w_{j, 0}$, we use Proposition 17, to see that

$$
\begin{aligned}
& w_{1,0}=\frac{A_{2,0}}{d}, \\
& w_{0,0}=\frac{A_{1,0}}{d}-\frac{A_{2,0}}{d^{2}} .
\end{aligned}
$$

From the definition of the traces, recalled in Definition 39 in "Appendix" (see also [3, p. 247]), we have that

$$
\begin{aligned}
& A_{2,0}=\operatorname{TR}\left(P^{-d}\right), \\
& A_{1,0}=d \cdot \widehat{\operatorname{TR}}_{x, \xi}\left(P^{-d}\right),
\end{aligned}
$$

which gives the claimed coefficients.

To prove Theorem 2 it suffices to prove that

$$
N(\lambda)=(N * \rho)(\lambda)+o\left(\lambda^{d-1} \log \lambda\right),
$$

where $(N * \rho)(\lambda)$ is obtained through Propositions 15 and 17 .

We define the microlocal return time function $\Pi: \mathcal{W} \rightarrow \mathbb{R}_{+} \cup\{\infty\}$ by

$$
\Pi(x, \xi)=\inf \left\{t>0: \exp \left(t \mathbf{X}_{\sigma(P)}\right)(x, \xi)=(x, \xi)\right\},
$$

and $\Pi(x, \xi)=\infty$ if no such $t$ exists. For a set $\Gamma \in \mathcal{W}$, we set $\Pi_{\Gamma}=\inf _{z \in \Gamma} \Pi(z)$.

We will need a microlocalized version of the Poisson relation.

Proposition 22. Let $\Gamma \subset \mathcal{W}$ and $\hat{\chi} \in \mathcal{C}_{c}^{\infty}(\mathbb{R})$ with $\operatorname{supp} \hat{\chi} \subset\left(0, \Pi_{\Gamma}\right)$. For all $B \in \mathrm{OpSG}_{\mathrm{cl}}^{0,0}$ with $\mathrm{WF}_{\mathrm{SG}}^{\prime}(B) \subset \Gamma$, we have that

$$
\hat{\chi}(t) \operatorname{Tr}\left(U(t) B B^{*}\right) \in \mathcal{C}_{c}^{\infty}(\mathbb{R}) .
$$

In particular, $\left(\chi * N_{B}^{\prime}\right)(\lambda) \in O\left(\lambda^{-\infty}\right)$.

The proof is a standard argument (cf. Wunsch [39]) and is only sketched here.

Proof of Proposition 22. For $t_{0} \in \operatorname{supp} \hat{\chi}$ and $(x, \xi) \in \Gamma$, we choose a conic neighbourhood $U$ of $(x, \xi)$ such that

$$
[\Phi(t) U] \cap U=\emptyset
$$

for all $t \in\left(t_{0}-\epsilon, t_{0}+\epsilon\right)$ with $\epsilon>0$ sufficiently small. The existence of this neighbourhood is guaranteed by the conditions on $\Gamma$ and supp $\hat{\chi}$. Choose $\tilde{B} \in \mathrm{Op} \mathrm{SG}_{\mathrm{cl}}^{0,0}$ with $\mathrm{WF}_{\mathrm{SG}}^{\prime}(\tilde{B}) \subset U$. Lemma 14 implies that for any $k \in \mathbb{N}$,

$$
\partial_{t}^{k}(\tilde{B} U(t) \tilde{B})=\tilde{B} P^{k} U(t) \tilde{B} \in \mathcal{L}\left(\mathcal{S}^{\prime}\left(\mathbb{R}^{d}\right), \mathcal{S}\left(\mathbb{R}^{d}\right)\right)
$$

hence $\tilde{B} U(t) \tilde{B}$ and all its derivatives are trace class. We obtain the claim by using a partition of unity. 
We also define the modified return time

$$
\tilde{\Pi}(x, \xi)=\max \{\Pi(x, \xi), \epsilon\},
$$

where $\epsilon$ is given as in (12), and set $\tilde{\Pi}_{\Gamma}=\inf _{z \in \Gamma} \tilde{\Pi}(z)$. The main tool to prove Theorem 2 is Proposition 23.

Proposition 23. It holds true that

$$
\limsup _{\lambda \rightarrow \infty} \frac{|N(\lambda)-(N * \rho)(\lambda)|}{\lambda^{d-1} \log \lambda} \leq C \int_{\mathcal{W}^{\psi} e} \tilde{\Pi}(x, \xi)^{-1} \frac{\mathrm{d} S}{p_{1,1}(x, \xi)} .
$$

Proof of Theorem 2. The claim follows immediately by Proposition 23, since the assumptions imply that $\Pi(x, \xi)^{-1}=0$ almost everywhere on $\mathcal{W}^{\psi e}$. From Proposition 19, we obtain the coefficient $\gamma_{0}$.

Proof of Proposition 23. Consider an open covering $\left\{\Gamma_{j}^{\bullet}\right\}$ of $\mathcal{W}$ with $\bullet \in\{\psi$, $e, \psi e\}$ and $j \in\left\{1, \cdots, n_{\bullet}\right\}$ such that $\Gamma_{j}^{\psi} \subset \mathcal{W}^{\psi}$ and $\Gamma_{j}^{e} \subset \mathcal{W}^{e}$ do not intersect $\mathcal{W}^{\psi e}$, and $\Gamma_{j}^{\psi e} \cap \mathcal{W}^{\psi e} \neq \emptyset$.

We consider a partition of unity on the level of operators such that

$$
I=\sum_{j=1}^{n_{\psi}} A_{j}^{\psi}\left(A_{j}^{\psi}\right)^{*}+\sum_{j=1}^{n_{e}} A_{j}^{e}\left(A_{j}^{e}\right)^{*}+\sum_{j=1}^{n_{\psi e}} A_{j}^{\psi e}\left(A_{j}^{\psi e}\right)^{*}+R,
$$

where $A_{j}^{\psi} \in \mathrm{OpSG}_{\mathrm{cl}}^{0,-\infty}, A_{j}^{e} \in \mathrm{OpSG}_{\mathrm{cl}}^{-\infty, 0}, A_{j}^{\psi e} \in \mathrm{OpSG}_{\mathrm{cl}}^{0,0}$, and $R \in \mathcal{L}\left(\mathcal{S}^{\prime}, \mathcal{S}\right)$. Furthermore, we assume that $\mathrm{WF}_{\mathrm{SG}}^{\prime}\left(A_{j}^{\bullet}\right) \subset \Gamma_{j}^{\bullet}$.

Inserting the partition of unity into the counting function yields

$$
N(\lambda)=\sum_{j=1}^{n_{\psi}} N_{j}^{\psi}(\lambda)+\sum_{j=1}^{n_{e}} N_{j}^{e}(\lambda)+\sum_{j=1}^{n_{\psi e}} N_{j}^{\psi e}(\lambda)+\operatorname{Tr}\left(E_{\lambda} R\right),
$$

where as before $N_{j}^{\bullet}(\lambda)=\operatorname{Tr} E_{\lambda} A_{j}^{\bullet}\left(A_{j}^{\bullet}\right)^{*}=\sum_{\lambda_{k}<\lambda}\left\|\left(A_{j}^{\bullet}\right)^{*} \psi_{k}\right\|^{2}$. Here, $\psi_{k}$ are the eigenfunctions of $P$ with eigenvalue $\lambda_{k}$.

Since $A_{j}^{e}$ and $A_{j}^{\psi e}$ have wavefront set near the boundary of $X$, we can choose local coordinates such that $A_{j}^{e}$ and $A_{j}^{\psi e}$ become SG-operators on $\mathbb{R}^{d}$ of order $(-\infty, 0)$ and $(0,0)$, respectively. As in the proof of Theorem 1 , the parametrix of the wave equation then is locally a smooth family of SG-Fourier integral operators.

The asymptotics for $N_{j}^{\psi}$ and $N_{j}^{e}$ are standard and follow from similar arguments as in Hörmander [18], exchanging the roles of variable and covariable for $N_{j}^{e}$. We can also adapt the proof of Proposition 15. By observing that for $N_{j}^{e}(\lambda)$ and $N_{j}^{\psi}(\lambda)$, the amplitude is supported only near one boundary face, we obtain

$$
\begin{aligned}
& \left(N_{j}^{e} * \rho\right)(\lambda) \sim \sum_{k=0}^{\infty} C_{e, j, k} \lambda^{d-k} \\
& \left(N_{j}^{\psi} * \rho\right)(\lambda) \sim \sum_{k=0}^{\infty} C_{\psi, j, k} \lambda^{d-k} .
\end{aligned}
$$


Using the standard Tauberian theorem [33, Theorem B.2.1] yields

$$
\begin{aligned}
& \left(N_{j}^{e} * \rho\right)(\lambda)=\left(N_{j}^{e} * \rho\right)(\lambda)+O\left(\lambda^{d-1}\right), \\
& \left(N_{j}^{\psi} * \rho\right)(\lambda)=\left(N_{j}^{\psi} * \rho\right)(\lambda)+O\left(\lambda^{d-1}\right) .
\end{aligned}
$$

The operator $E_{\lambda} R$ is regularising; thus, its trace is uniformly bounded. We arrive at

$$
N(\lambda)-(N * \rho)(\lambda)=\sum_{j=1}^{n_{\psi e}}\left[N_{j}^{\psi e}(\lambda)-\left(N_{j}^{\psi e} * \rho\right)(\lambda)\right]+O\left(\lambda^{d-1}\right) .
$$

It remains to estimate the terms $N_{j}^{\psi e}(\lambda)-\left(N_{j}^{\psi e} * \rho\right)(\lambda)$. For this, let

$$
\Pi_{j}=\inf _{(x, \xi) \in \Gamma_{j}^{\psi e}} \Pi(x, \xi), \quad \tilde{\Pi}_{j}=\max \left\{\Pi_{j}, \epsilon\right\} .
$$

For $1 / T<\epsilon$, we have by Proposition 15 that

$$
\left(N_{j}^{\psi e} * \rho\right)(\lambda) \sim \sum_{k=0}^{\infty} \sum_{j=0,1} w_{j k} \lambda^{d-k}(\log \lambda)^{j} .
$$

This implies that the derivative is given by

$$
\left(\partial_{\lambda} N_{j}^{\psi e} * \rho\right)(\lambda)=d \cdot w_{1,0} \lambda^{d-1} \log \lambda+O\left(\lambda^{d-1}\right),
$$

where $w_{1,0}$ is given by Proposition 18. Namely,

$$
w_{1,0}=\frac{1}{d} \int_{\mathbb{S}^{d-1}} \int_{\mathbb{S}^{d-1}}\left[p_{\psi e}(\theta, \omega)\right]^{-d} \cdot\left|\sigma^{\psi e}\left(A_{j}^{\psi e}\right)(\theta, \omega)\right|^{2} \mathrm{~d} \theta \mathrm{d} \omega .
$$

Together with Proposition 22, this implies that

$$
\begin{aligned}
\left(N_{j}^{\psi e} * \rho_{T}\right)(\lambda) & =\left(N_{j}^{\psi e} * \rho\right)(\lambda)+O\left(\lambda^{-\infty}\right) \\
& =\sum_{k=0}^{\infty} \sum_{j=0,1} w_{j k} \lambda^{d-k}(\log \lambda)^{j}+O\left(\lambda^{-\infty}\right)
\end{aligned}
$$

for $1 / T<\tilde{\Pi}_{j}$.

Applying the Tauberian theorem to $N_{j}^{\psi e} * \rho_{T}$ yields

$$
\begin{aligned}
& \frac{\left|N_{j}^{\psi e}(\lambda)-\left(N_{j}^{\psi e} * \rho\right)(\lambda)\right|}{\lambda^{d-1} \log \lambda} \\
& \quad \lesssim \tilde{\Pi}_{j}^{-1} \int_{\mathbb{S}^{d-1}} \int_{\mathbb{S}^{d-1}}\left|\sigma^{\psi e}\left(A_{j}^{\psi e}\right)(\omega, \theta)\right|^{2} p_{1,1}(\omega, \theta)^{-d} \mathrm{~d} \theta \mathrm{d} \omega
\end{aligned}
$$

for $\lambda \geq \tilde{\Pi}_{j}$. Taking the limsup and summing over all $j$ gives

$$
\begin{aligned}
& \limsup _{\substack{\lambda \rightarrow \infty \\
n_{\psi e}}} \frac{|N(\lambda)-(N * \rho)(\lambda)|}{\lambda^{d-1} \log \lambda} \\
& \lesssim \sum_{j=1}^{-1} \int_{\mathbb{I}^{d-1}} \int_{\mathbb{S}^{d-1}}\left|\sigma^{\psi e}\left(A_{j}^{\psi e}\right)(\omega, \theta)\right|^{2} p_{1,1}(\omega, \theta)^{-d} \mathrm{~d} \theta \mathrm{d} \omega .
\end{aligned}
$$

The right-hand side is an upper Riemann sum; therefore, we obtain the claim by shrinking the partition of unity. 


\section{An Example: The Model Operator $\boldsymbol{P}=\langle\cdot\rangle\langle D\rangle$}

In this section, we will consider the case of the operator $P=\langle\cdot\rangle\langle D\rangle$ on $\mathbb{R}^{d}$. First, we compute the full symbol of $P$ near the corner:

$$
\begin{aligned}
\langle x\rangle\langle\xi\rangle & =|x| \cdot|\xi| \cdot\left(1+\frac{1}{|x|^{2}}\right)^{\frac{1}{2}}\left(1+\frac{1}{|\xi|^{2}}\right)^{\frac{1}{2}} \\
& =|x| \cdot|\xi| \cdot \sum_{j, k=0}^{\infty}\left(\begin{array}{c}
\frac{1}{2} \\
j
\end{array}\right)\left(\begin{array}{c}
\frac{1}{2} \\
k
\end{array}\right)(-1)^{j+k} \frac{1}{|x|^{2 j} \cdot|\xi|^{2 k}} \\
& =\sum_{j, k=0}^{\infty}\left(\begin{array}{c}
\frac{1}{2} \\
j
\end{array}\right)\left(\begin{array}{c}
\frac{1}{2} \\
k
\end{array}\right)(-1)^{j+k}|x|^{1-2 j} \cdot|\xi|^{1-2 k} .
\end{aligned}
$$

It follows that $p_{\psi e}(x, \xi)=\sigma^{\psi e}(P)(x, \xi)=|x||\xi|, p_{\psi}(x, \xi)=|\xi|\langle x\rangle$, and $p_{e}(x, \xi)=|x|\langle\xi\rangle$.

We have to investigate the flow of the principal symbol $p_{\psi e}$ in the corner. The Hamiltonian vector field on $\mathbb{R}^{2 d}$ is given by

$$
\mathrm{X}_{p_{\psi e}}=\partial_{\xi} p_{\psi e} \partial_{x}-\partial_{x} p_{\psi e} \partial_{\xi}
$$

First, we show that the angle between $x$ and $\xi$ is invariant under the flow. This follows from

$$
\begin{aligned}
\partial_{t}\langle x, \xi\rangle & =\left\langle\partial_{t} x, \xi\right\rangle+\left\langle x, \partial_{t} \xi\right\rangle \\
& =\frac{|x|}{|\xi|}\langle\xi, \xi\rangle-\frac{|\xi|}{|x|}\langle x, x\rangle \\
& =|x||\xi|-|x||\xi|=0 .
\end{aligned}
$$

Hence, the quantity

$$
c=c\left(x_{0}, \xi_{0}\right)=\frac{\left\langle x_{0}, \xi_{0}\right\rangle}{\left|x_{0}\right|\left|\xi_{0}\right|}
$$

is preserved by the flow. The Hamiltonian flow $\Phi^{\psi e}(t): \mathcal{W}^{\psi e} \rightarrow \mathcal{W}^{\psi e}$ is given by the angular part.

Lemma 24. The differential equation for $\omega=x /|x|$ and $\theta=\xi /|\xi|$ describing the Hamiltonian flow $\Phi^{\psi e}(t): \mathcal{W}^{\psi e} \rightarrow \mathcal{W}^{\psi e}$ is given by

$$
\left\{\begin{aligned}
\partial_{t} \omega & =-c \omega+\theta \\
\partial_{t} \theta & =-\omega+c \theta
\end{aligned}\right.
$$

Proof. We observe that

$$
\partial_{t} \frac{x(t)}{|x(t)|}=\frac{\partial_{t} x(t)}{|x(t)|}-\frac{x(t) \partial_{t}|x(t)|}{|x(t)|^{2}} .
$$

The calculation of $\partial_{t}|x|$ is straightforward:

$$
\partial_{t}|x|=\frac{\langle x, \xi\rangle}{|x||\xi|} \cdot|x|=\frac{\left\langle x_{0}, \xi_{0}\right\rangle}{\left|x_{0}\right|\left|\xi_{0}\right|} \cdot|x|
$$


This implies

$$
\partial_{t} \frac{x(t)}{|x(t)|}=\frac{\xi(t)}{|\xi(t)|}-c \frac{x(t)}{|x(t)|},
$$

as claimed. The second equation follows likewise.

Proposition 25. The return time function $\Pi: \mathcal{W}^{\psi e} \rightarrow \mathbb{R}$ is given by

$$
\Pi(\omega, \theta)= \begin{cases}\frac{2 \pi}{\sqrt{1-\langle\omega, \theta\rangle^{2}}}, & \langle\omega, \theta\rangle^{2} \neq 1 \\ 0, & \langle\omega, \theta\rangle^{2}=1 .\end{cases}
$$

Proof. The system of differential equations (20) decomposes into $d$ decoupled systems of the form

$$
\partial_{t} v(t)=A v(t)
$$

where

$$
A=\left(\begin{array}{ll}
-c & 1 \\
-1 & c
\end{array}\right)
$$

We note that the eigenvalues of the matrix $A$ are given by $\lambda_{ \pm}=$ $\pm i \sqrt{1-c^{2}}$. Thus, we have that the fundamental solution to the differential equation $(20)$ for $(\omega, \theta)$ is given by

$$
S \cdot\left(\begin{array}{cc}
\mathrm{e}^{-i t \sqrt{1-c^{2}}} & \mathrm{I}_{d} \\
0 & \mathrm{e}^{i t \sqrt{1-c^{2}}} \mathrm{I}_{d}
\end{array}\right) \cdot S^{-1}
$$

for some unitary matrix $S=S(c)$. The claim follows by choosing the minimal $t>0$ with $t \sqrt{1-c^{2}} \in 2 \pi \mathbb{Z}$ and noting that $c=\langle\omega(0), \theta(0)\rangle=\left\langle\omega_{0}, \theta_{0}\right\rangle$ for $\omega_{0}, \theta_{0} \in \mathbb{S}^{d-1}$.

Remark 26. Proposition 25 shows that Theorem 2 cannot be applied to $P$. Nevertheless, we calculate $\gamma_{0}$ in Proposition 28.

Proof of Theorem 6. By the Weyl law, Theorem 1, we have that

$$
N(\lambda)=\gamma_{2} \lambda^{d} \log \lambda+\gamma_{1} \lambda^{d}+O\left(\lambda^{d-1} \log \lambda\right) .
$$

So it remains to calculate the corresponding Laurent coefficients of $\zeta(s)$. By Proposition 40, we have

$$
\begin{aligned}
\gamma_{2} & =\frac{\operatorname{TR}\left(P^{-d}\right)}{d}=\frac{\operatorname{Tr}_{\psi, e}\left(P^{-d}\right)}{d}=\frac{(2 \pi)^{-d}}{d} \int_{\mathbb{S}^{d-1}} \int_{\mathbb{S}^{d-1}} p_{\psi e}(\theta, \omega)^{-d} \mathrm{~d} \theta \mathrm{d} \omega \\
& =\frac{(2 \pi)^{-d}}{d} \int_{\mathbb{S}^{d-1}} \int_{\mathbb{S}^{d-1}} \mathrm{~d} \theta \mathrm{d} \omega \\
& =\frac{\left[\operatorname{vol}\left(\mathbb{S}^{d-1}\right)\right]^{2}}{(2 \pi)^{d}} \frac{1}{d} .
\end{aligned}
$$


Again by Proposition 40,

$$
\begin{aligned}
\gamma_{1} & =\widehat{\mathrm{TR}}_{x, \xi}\left(P^{-d}\right)-\frac{\operatorname{TR}\left(P^{-d}\right)}{d^{2}} \\
& =\frac{1}{d}\left(\frac{1}{d} \widehat{\operatorname{TR}}_{\theta}\left(P^{-d}\right)+\widehat{\operatorname{Tr}}_{\psi}\left(P^{-d}\right)+\widehat{\operatorname{Tr}}_{e}\left(P^{-d}\right)-\frac{1}{d} \operatorname{TR}\left(P^{-d}\right)\right)
\end{aligned}
$$

First, we note that $\widehat{\mathrm{TR}}_{\psi}\left(P^{-d}\right)=\widehat{\mathrm{TR}}_{e}\left(P^{-d}\right)$ and the last term we already calculated for $\gamma_{2}$. We recall that $p_{\psi e}=1$ on $\mathbb{S}^{d-1} \times \mathbb{S}^{d-1}$. Thus, we have for $\widehat{\mathrm{TR}}_{\theta}\left(P^{-d}\right)$ that

$$
\begin{aligned}
\widehat{\mathrm{TR}}_{\theta}\left(P^{-d}\right) & =\frac{1}{(2 \pi)^{d}} \int_{\mathbb{S}^{d-1}} \int_{\mathbb{S}^{d-1}} p_{\psi e}(\theta, \omega)^{-d} \log \left(p_{\psi e}(\theta, \omega)^{-d}\right) \mathrm{d} \theta \mathrm{d} \omega \\
& =0
\end{aligned}
$$

This implies

$$
\gamma_{1}=\frac{2 \cdot \widehat{\mathrm{TR}}_{e}\left(P^{-d}\right)}{d}-\frac{\mathrm{TR}\left(P^{-d}\right)}{d^{2}}
$$

Hence, we only have to calculate $\widehat{\mathrm{TR}}_{e}\left(P^{-d}\right)$ :

$$
\begin{aligned}
\widehat{\mathrm{TR}}_{e}\left(P^{-d}\right)= & \frac{1}{(2 \pi)^{d}} \lim _{\tau \rightarrow+\infty}\left\{\int_{\mathbb{S}^{d-1}} \int_{|\xi| \leq \tau} p_{e}(\theta, \xi)^{-d} \mathrm{~d} \theta \mathrm{d} \xi\right. \\
& \left.-(\log \tau) \int_{\mathbb{S}^{d-1}} \int_{\mathbb{S}^{d-1}} p_{\psi e}(\theta, \omega)^{-d} \mathrm{~d} \theta \mathrm{d} \omega\right\} \\
= & \frac{\operatorname{vol}\left(\mathbb{S}^{d-1}\right)^{2}}{(2 \pi)^{d}} \lim _{\tau \rightarrow+\infty}\left[\operatorname{vol}\left(\mathbb{S}^{d-1}\right)^{-1} \int_{|x| \leq \tau}\langle x\rangle^{-d} \mathrm{~d} x-\log \tau\right] .
\end{aligned}
$$

Using polar coordinates, we see that

$$
\operatorname{vol}\left(\mathbb{S}^{d-1}\right)^{-1} \int_{|x| \leq \tau}\langle x\rangle^{-d} \mathrm{~d} x=\int_{0}^{\tau}\left(1+r^{2}\right)^{-d / 2} r^{d-1} \mathrm{~d} r .
$$

Now, we perform a change of variables $r=t^{-\frac{1}{2}} \Leftrightarrow t=r^{-2}>0$, so that

$$
\begin{aligned}
\int_{0}^{\tau}\left(1+r^{2}\right)^{-\frac{d}{2}} r^{d-1} \mathrm{~d} r & =\frac{1}{2} \int_{\tau^{-2}}^{+\infty}(t+1)^{-\frac{d}{2}} t^{-1} \mathrm{~d} t \\
& =\frac{1}{2} \int_{\tau^{-2}}^{+\infty} \frac{\mathrm{d} t}{t(t+1)}-\frac{1}{2} \int_{\tau^{-2}}^{+\infty}\left[(1+t)^{-1}-(t+1)^{-\frac{d}{2}}\right] \frac{\mathrm{d} t}{t} .
\end{aligned}
$$

For $\operatorname{Re} z>0$, we have that (cf. [16, \#8.36])

$$
\Psi(z)=\int_{0}^{+\infty}\left[(1+t)^{-1}-(t+1)^{-z}\right] \frac{\mathrm{d} t}{t}-\gamma,
$$


where $\Psi(z)$ is the digamma function, defined by (3). By elementary computations, we obtain

$$
\begin{aligned}
\int_{\tau^{-2}}^{+\infty} \frac{\mathrm{d} t}{t(t+1)}+\log \tau^{-2}= & \lim _{\kappa \rightarrow+\infty}\left[\log \frac{\kappa}{\kappa+1}-\log \tau^{-2}+\log \left(1+\tau^{-2}\right)\right] \\
& +\log \tau^{-2} \\
= & \log \left(1+\tau^{-2}\right) \longrightarrow 0 \text { for } \tau \rightarrow+\infty
\end{aligned}
$$

Hence, we have that

$$
\begin{aligned}
\lim _{\tau \rightarrow+\infty} & {\left[\operatorname{vol}\left(\mathbb{S}^{d-1}\right)^{-1} \int_{|x| \leq \tau}\langle x\rangle^{-d} \mathrm{~d} x-\log \tau\right] } \\
= & \frac{1}{2} \lim _{\tau \rightarrow \infty}\left[\int_{\tau^{-2}}^{+\infty} \frac{\mathrm{d} t}{t(t+1)}+\log \tau^{-2}\right] \\
& -\frac{1}{2} \lim _{\tau \rightarrow \infty} \int_{\tau^{-2}}^{+\infty}\left[(1+t)^{-1}-(t+1)^{-\frac{d}{2}}\right] \frac{\mathrm{d} t}{t} \\
= & -\frac{1}{2}[\Psi(d / 2)+\gamma] .
\end{aligned}
$$

Summing up, we have obtained

$$
\gamma_{1}=-\frac{\left[\operatorname{vol}\left(\mathbb{S}^{d-1}\right)\right]^{2}}{(2 \pi)^{d}} \frac{1}{d} \cdot\left[\Psi\left(\frac{d}{2}\right)+\gamma+\frac{1}{d}\right] .
$$

The proof is complete.

Remark 2\%. Using the properties of the function $\Psi$, we can make (22) more explicit. Indeed (see, for example, [16, \#8.366, page 945]), we find:

$$
\gamma_{1}= \begin{cases}-\frac{\left[\operatorname{vol}\left(\mathbb{S}^{d-1}\right)\right]^{2}}{(2 \pi)^{d}} \frac{1}{d}\left(\frac{1}{d}+2 \sum_{k=1}^{\frac{d-1}{2}} \frac{1}{2 k-1}-2 \log 2\right), & \text { if } d \text { is odd, } \\ -\frac{\left[\operatorname{vol}\left(\mathbb{S}^{d-1}\right)\right]^{2}}{(2 \pi)^{d}} \frac{1}{d}\left(\frac{1}{d}+\sum_{k=1}^{\frac{d}{2}-1} \frac{1}{k}\right), & \text { if } d \text { is even. }\end{cases}
$$

In particular, we have that

$$
\gamma_{1}= \begin{cases}-\frac{2}{\pi}(1-2 \log 2), & d=1 \\ -\frac{1}{4}, & d=2 .\end{cases}
$$

Even though the coefficient $\gamma_{0}$ does not appear in the Weyl law of $P$, for the sake of completeness we show that it is not hard to compute in this case.

Proposition 28. For the operator $P=\langle\cdot\rangle\langle D\rangle$, we have that $\gamma_{0}=0$.

Proof. To calculate $\gamma_{0}$, we switch to Weyl quantization. Letting $p^{w}(x, D)=P$, the symbol $p$ has an asymptotic expansion

$$
p=p_{\psi e}+p_{0,0} \quad \bmod \mathrm{SG}^{-1,0}+\mathrm{SG}^{0,-1},
$$


where $p_{0,0}(x, \xi)=\frac{\langle x, \xi\rangle}{|x||\xi|}$ is the subprincipal symbol of $p$. Hence, for the symbol of $P^{d-1}=\left[a^{w}(x, D)\right]^{d-1}$ we find an asymptotic expansion

$$
p^{d-1}=\left(p_{\psi e}\right)^{d-1}+(d-1)\left(p_{\psi e}\right)^{d-2} p_{0,0} \bmod \mathrm{SG}^{d-3, d-2}+\mathrm{SG}^{d-2, d-3} .
$$

This implies for the symbol $p^{-d+1}$ of the inverse of $\left[p^{w}(x, D)\right]^{d-1}$ that

$$
p^{-d+1}=\left(p_{\psi e}\right)^{-d+1}-(d-1) \frac{p_{0,0}}{\left(p_{\psi e}\right)^{d}} \bmod \mathrm{SG}^{-d-1,-d}+\mathrm{SG}^{-d,-d-1} .
$$

Therefore, by Propositions 17 and 19, we have that

$$
\begin{aligned}
\gamma_{0} & =\frac{1}{d-1} A_{2,1} \\
& =-(2 \pi)^{-d} \int_{\mathbb{S}^{d-1}} \int_{\mathbb{S}^{d-1}} \frac{p_{0,0}}{\left(p_{\psi e}\right)^{d}} \mathrm{~d} \theta \mathrm{d} \omega \\
& =-(2 \pi)^{-d} \int_{\mathbb{S}^{d-1}} \int_{\mathbb{S}^{d-1}}\langle\theta, \omega\rangle \mathrm{d} \theta \mathrm{d} \omega=0 .
\end{aligned}
$$

\section{Acknowledgements}

We would like to thank R. Schulz for many helpful discussions and various remarks on the manuscript. We are grateful to the anonymous Referees, for their comments, suggestions and constructive criticism.

Funding Open access funding provided by Università degli Studi di Torino within the CRUI-CARE Agreement.

Open Access. This article is licensed under a Creative Commons Attribution 4.0 International License, which permits use, sharing, adaptation, distribution and reproduction in any medium or format, as long as you give appropriate credit to the original author(s) and the source, provide a link to the Creative Commons licence, and indicate if changes were made. The images or other third party material in this article are included in the article's Creative Commons licence, unless indicated otherwise in a credit line to the material. If material is not included in the article's Creative Commons licence and your intended use is not permitted by statutory regulation or exceeds the permitted use, you will need to obtain permission directly from the copyright holder. To view a copy of this licence, visit http://creativecommons.org/ licenses/by/4.0/.

Publisher's Note Springer Nature remains neutral with regard to jurisdictional claims in published maps and institutional affiliations. 


\section{Appendix A. Classical Operators on Asymptotically Euclidean Manifolds}

\section{A.1. Scattering Geometry}

We refer the reader to, for example, $[7,28]$ for a detailed study of scattering geometry and recall here only a few definitions and notation.

Definition 29. An asymptotically Euclidean manifold $(X, g)$ is a compact manifold with boundary $X$, whose interior is equipped with a Riemannian metric $g$ that is supposed to take the form, in a tubular neighbourhood of the boundary,

$$
g=\frac{\mathrm{d} \rho_{X}^{2}}{\rho_{X}^{4}}+\frac{g_{\partial}}{\rho_{X}^{2}},
$$

where $\rho_{X}$ is a boundary defining function and $g_{\partial} \in \mathcal{C}^{\infty}\left(X, \operatorname{Sym}^{2} T^{*} X\right)$ restricts to a metric on $\partial X$.

We set $\mathbb{B}^{d}=\left\{x \in \mathbb{R}^{d}:|x| \leq 1\right\}$ and denote $\partial \mathbb{B}^{d}=\mathbb{S}^{d-1},\left(\mathbb{B}^{d}\right)^{o}=\{x \in$ $\left.\mathbb{R}^{d}:|x|<1\right\}$, and $\mathbb{R}_{+}=(0, \infty)$. Pick any diffeomorphism $\iota: \mathbb{R}^{d} \rightarrow\left(\mathbb{B}^{d}\right)^{o}$ that for $|x|>3$ is given by

$$
\iota: x \mapsto \frac{x}{|x|}\left(1-\frac{1}{|x|}\right) .
$$

Then, its inverse is given, for $|y| \geq \frac{2}{3}$, by

$$
\iota^{-1}: y \mapsto \frac{y}{|y|}(1-|y|)^{-1} .
$$

The map $\iota$ is called the radial compactification map. The associated polar coordinates equip $\mathbb{R}^{d}$ with a differential structure "at infinity". Indeed, introducing polar coordinates $(r, \varphi) \in \mathbb{R}^{d}$ we see that $\iota$ is simply given (for large $r$ ) by

$$
r \mapsto 1-\frac{1}{r} \quad \text { and } \quad \varphi \mapsto \varphi
$$

Denote by $x \mapsto[x]$ any smooth function $\mathbb{R}^{d} \rightarrow \mathbb{R}_{+}$that coincides with $|x|$ for $|x|>3$. Then, the map $\mathbb{B}^{d} \rightarrow[0, \infty)$ given by $y \mapsto \frac{1}{\left[\iota^{-1}(y)\right]}=: \rho_{\mathbb{B}^{d}}$ is a boundary defining function for $\mathbb{B}^{d}$. Notice that, for $|y|>2 / 3$, the map $y \mapsto \rho_{\mathbb{B}^{d}}$ is simply given by $y \mapsto(1-|y|)$. In a collar neighbourhood of the boundary, $0 \leq \rho_{\mathbb{B}^{d}}<1 / 3$, the metric induced by these coordinates from the standard Euclidean metric on $\mathbb{R}^{d}$ is given by

$$
g=\frac{\mathrm{d} \rho_{\mathbb{B}^{d}}^{2}}{\rho_{\mathbb{B}^{d}}^{2}}+\frac{g_{\mathbb{S}^{d-1}}}{\rho_{\mathbb{B}^{d}}^{4}},
$$

where $g_{\mathbb{S}^{d-1}}$ is the (lifted) standard metric on the $(d-1)$-sphere.

For any compact manifold with boundary $X$ with boundary defining function $\rho_{X}$, we define the space of scattering vector fields ${ }^{\mathrm{sc}} \mathcal{V}(x):=\rho_{X}{ }^{b} \mathcal{V}(X)$, where ${ }^{b} \mathcal{V}(X)$ is the space of tangential vector fields. There is a natural vector bundle, ${ }^{\mathrm{sc}} T X$ such that the sections of ${ }^{\mathrm{sc}} T X$ are exactly the scattering vector fields. The dual bundle is the scattering cotangent bundle, ${ }^{\mathrm{sc}} T^{*} X$. Using the 
fibrewise radial compactification, we obtain a manifold with corners ${ }^{\mathrm{sc}} \bar{T}^{*} X$ with boundary defining functions $\rho_{X}$ and $\rho_{\Xi}$.

The new-formed fibre boundary may be identified with a rescaling of the cosphere bundle, called ${ }^{\mathrm{sc}} S^{*} X$. Since $X$ is a compact manifold with boundary, ${ }^{\mathrm{sc}} \bar{T}^{*} X$ is a compact manifold with corners. The boundary $\mathcal{W}$ of ${ }^{\mathrm{sc}} \bar{T}^{*} X$ splits into three components:

$$
\mathcal{W}^{e}:={ }^{\mathrm{sc}} T_{\partial X}^{*} X, \quad \mathcal{W}^{\psi}:={ }^{\mathrm{sc}} S_{X^{\circ}}^{*} X, \quad \mathcal{W}^{\psi e}:={ }^{\mathrm{sc}} S_{\partial X}^{*} X .
$$

It can be shown (cf. [7, Section 1], in particular Example 1.14) that, under the above identification of $\mathbb{R}^{d}$ with the interior of $\mathbb{B}^{d}$, the SG-classical symbols spaces $\mathrm{SG}_{\mathrm{cl}}^{m_{\psi}, m_{e}}\left(\mathbb{R}^{d}\right)$ become $\rho_{X}^{-m_{e}} \rho_{\Xi}^{-m_{\psi}} \mathcal{C}^{\infty}\left(\mathbb{B}^{d} \times \mathbb{B}^{d}\right)$.

Let $X$ be an asymptotically Euclidean manifold, the SG-classical symbols of order $m_{\psi}, m_{e} \in \mathbb{R}$ are given by

$$
\mathrm{SG}_{\mathrm{cl}}^{m_{\psi}, m_{e}}(X)=\rho_{X}^{-m_{e}} \rho_{\Xi}^{-m_{\psi}} \mathcal{C}^{\infty}\left({ }^{\mathrm{sc}} \bar{T}^{*} X\right)
$$

Using local coordinates, one can define SG-operators, which is denoted by $\mathrm{OpSG}_{\mathrm{cl}}(X)$, on general asymptotically Euclidean manifolds, see Melrose [27, Definition 3]. ${ }^{4}$

Let $a \in \rho_{X}^{-m_{e}} \rho_{\Xi}^{-m_{\psi}} \mathcal{C}^{\infty}\left(\mathrm{sc}_{T^{*}}^{*}\right)$, and set $f=\rho_{X}^{m_{e}} \rho_{\Xi}^{m_{\psi}} a$. The symbols $\sigma^{\bullet}(a)$ of $a$ at a point $p=\left(\rho_{X}, x, \rho_{\Xi}, \xi\right) \in \mathcal{W}^{\bullet}$ are defined by

$$
\begin{aligned}
\sigma^{\psi}(a)\left(\rho_{X}, x, \rho_{\Xi}, \xi\right) & =\rho_{X}^{-m_{e}} \rho_{\Xi}^{-m_{\psi}} f\left(\rho_{X}, x, 0, \xi\right), & & p \in \mathcal{W}^{\psi}, \\
\sigma^{e}(a)\left(\rho_{X}, x, \rho_{\Xi}, \xi\right) & =\rho_{X}^{-m_{e}} \rho_{\Xi}^{-m_{\psi}} f\left(0, x, \rho_{\Xi}, \xi\right), & & p \in \mathcal{W}^{e}, \\
\sigma^{\psi e}(a)\left(\rho_{X}, x, \rho_{\Xi}, \xi\right) & =\rho_{X}^{-m_{e}} \rho_{\Xi}^{-m_{\psi}} f(0, x, 0, \xi), & & p \in \mathcal{W}^{\psi e} .
\end{aligned}
$$

The principal symbol $\sigma(a) \in \mathcal{C}^{\infty}(\mathcal{W})$ is the triple

$$
\sigma(a)=\left(\sigma^{\psi}(a), \sigma^{e}(a), \sigma^{\psi e}(a)\right)
$$

A symbol $a \in \rho_{X}^{-m_{e}} \rho_{\Xi}^{-m_{\psi}} \mathcal{C}^{\infty}\left(\operatorname{sc}^{*} \bar{T}^{*} X\right)$ is called elliptic at $p \in \mathcal{W}$ if $\sigma(a)(p) \neq 0$ (cf. [26, Section 6.4]).

Melrose and Zworski [29] defined, for $a \in \rho_{X}^{-m_{e}} \rho_{\Xi}^{-m_{\psi}} \mathcal{C}^{\infty}\left({ }^{\mathrm{sc}} \bar{T}^{*} X\right)$, the Hamiltonian vector field

$$
{ }^{\mathrm{sc}} \mathrm{X}_{a} \in \rho_{X}^{-m_{e}+1} \rho_{\Xi}^{-m_{\psi}+1}{ }^{b} \mathcal{V}\left({ }^{\mathrm{sc}} \bar{T}^{*} X\right),
$$

which generalizes the usual Hamiltonian vector field to the compactified scattering cotangent bundle of asymptotically Euclidean manifolds.

For $a \in \rho_{X}^{-1} \rho_{\Xi}^{-1} \mathcal{C}^{\infty}\left({ }^{\mathrm{sc}} \bar{T}^{*} X\right)$, the Hamiltonian vector field is tangential to the boundary, and hence, its flow $\exp \left(t^{\mathrm{sc}} \mathrm{X}_{f}\right)$ can be restricted to a map

$$
\left.\exp \left(t^{\mathrm{sc}} \mathrm{X}_{a}\right)\right|_{\mathcal{W}}: \mathcal{W} \rightarrow \mathcal{W}
$$

that preserves the components $\mathcal{W}^{e}, \mathcal{W}^{\psi}$, and $\mathcal{W}^{\psi e}$. Note that the flow $t \mapsto$ $\left.\exp \left(t^{\mathrm{sc}} \mathbf{X}_{a}\right)\right|_{\mathcal{W}}$ depends only on the principal symbol $\sigma(a)$ of $a$.

\footnotetext{
${ }^{4}$ Note that Melrose uses the stereographic projection to compactify $\mathbb{R}^{d}$ to $\mathbb{S}_{+}^{d}$, but it was shown in [7, Remark 1.3] that these two compactifications are equivalent. Moreover, the space $\mathrm{Op} \mathrm{SG}_{\mathrm{cl}}$ is denoted by $\Psi_{\mathrm{sc}}$.
} 


\section{A.2. The Calculus of SG-Fourier Integral Operators}

The calculus of Fourier integral operators defined by means of general SGsymbols was initially studied in [5] and subsequently applied to the analysis of the corresponding hyperbolic problems in [6]. Their SG-classical counterparts have been considered in [11]. The theory was expanded along the years, and such operator class has been studied and employed also by other authors. Some of the most recent developments in this field have been obtained in the series of papers $[7,12,13]$ (see also [8] and the references quoted there). In this section, we recall some basic elements of the calculus of SG Fourier integral operators on $\mathbb{R}^{d}$ that are involved in the proof of the main results of this paper, relying on materials appeared, for example, in [1] (see [8] for more general classes of Fourier integral operators of SG type). Here, we write $A \asymp B$ when $A \lesssim B$ and $B \lesssim A$, where $A \lesssim B$ means that $A \leq c \cdot B$, for a suitable constant $c>0$. We will also write FIO for Fourier integral operator.

Definition 30. A real-valued function $\varphi \in \mathcal{C}^{\infty}\left(\mathbb{R}^{2 d}\right)$ belongs to the class $\mathcal{P}$ of SG phase functions if it satisfies the following conditions:

1. $\varphi \in \mathrm{SG}^{1,1}\left(\mathbb{R}^{2 d}\right)$;

2. $\left\langle\varphi_{x}^{\prime}(x, \xi)\right\rangle \asymp\langle\xi\rangle$ as $|(x, \xi)| \rightarrow \infty$;

3. $\left\langle\varphi_{\xi}^{\prime}(x, \xi)\right\rangle \asymp\langle x\rangle$ as $|(x, \xi)| \rightarrow \infty$.

Functions of class $\mathcal{P}$ are those used in the construction of the SG FIOs calculus. The SG FIOs of type I and type II, $\mathrm{Op}_{\varphi}(a)$ and $\mathrm{Op}_{\varphi}^{*}(b)$, are defined as

$$
u \mapsto\left(\mathrm{Op}_{\varphi}(a) u\right)(x)=(2 \pi)^{-n} \int \mathrm{e}^{i \varphi(x, \xi)} a(x, \xi) \widehat{u}(\xi) d \xi,
$$

and

$$
u \mapsto\left(\mathrm{Op}_{\varphi}^{*}(a) u\right)(x)=(2 \pi)^{-n} \iint \mathrm{e}^{i(x \xi-\varphi(y, \xi))} \overline{a(y, \xi)} u(y) \mathrm{d} y \mathrm{~d} \xi,
$$

respectively, with $\varphi \in \mathcal{P}$ and $a, b \in \mathrm{SG}^{m_{\psi}, m_{e}}, u \in \mathcal{S}$. Operators of type I and type II with the same phase function and symbol are formal $L^{2}$-adjoint of each other. Both operators of type I and type II are linear and continuous on $\mathcal{S}$, extendable to linear continuous operators on $\mathcal{S}^{\prime}$.

Theorem 31 about composition between SG pseudodifferential operators and SG FIOs was originally proved in $[5]$, see also $[8,11]$.

Theorem 31. Let $\varphi \in \mathcal{P}$ and assume $p \in \mathrm{SG}^{s_{\psi}, s_{e}}\left(\mathbb{R}^{2 d}\right), a, b \in \mathrm{SG}^{m_{\psi}, m_{e}}\left(\mathbb{R}^{2 d}\right)$. Then,

$$
\begin{aligned}
& \mathrm{Op}(p) \circ \mathrm{Op}_{\varphi}(a)=\mathrm{Op}_{\varphi}\left(c_{1}+r_{1}\right)=\mathrm{Op}_{\varphi}\left(c_{1}\right) \bmod \mathrm{OpSG}^{-\infty,-\infty}\left(\mathbb{R}^{d}\right), \\
& \mathrm{Op}(p) \circ \mathrm{Op}_{\varphi}^{*}(b)=\mathrm{Op}_{\varphi}^{*}\left(c_{2}+r_{2}\right)=\mathrm{Op}_{\varphi}^{*}\left(c_{2}\right) \bmod \mathrm{OpSG}^{-\infty,-\infty}\left(\mathbb{R}^{d}\right), \\
& \mathrm{Op}_{\varphi}(a) \circ \mathrm{Op}(p)=\mathrm{Op}_{\varphi}\left(c_{3}+r_{3}\right)=\mathrm{Op}_{\varphi}\left(c_{3}\right) \bmod \mathrm{OpSG}^{-\infty,-\infty}\left(\mathbb{R}^{d}\right), \\
& \mathrm{Op}_{\varphi}^{*}(b) \circ \mathrm{Op}(p)=\mathrm{Op}_{\varphi}^{*}\left(c_{4}+r_{4}\right)=\mathrm{Op}_{\varphi}^{*}\left(c_{4}\right) \bmod \mathrm{OpSG}^{-\infty,-\infty}\left(\mathbb{R}^{d}\right),
\end{aligned}
$$

for some $c_{j} \in \mathrm{SG}^{m_{\psi}+s_{\psi}, m_{e}+s_{e}}\left(\mathbb{R}^{2 d}\right), r_{j} \in \mathrm{SG}^{-\infty,-\infty}\left(\mathbb{R}^{2 d}\right), j=1, \ldots, 4$. 
To obtain the composition of SG FIOs of type I and type II, some more hypotheses are needed, leading to the definition of the class $\mathcal{P}_{r}$ of regular SG phase functions.

Definition 32. Let $r>0$. A function $\varphi \in \mathcal{P}$ belongs to the class $\mathcal{P}_{r}$ if it satisfies, for all $(x, \xi) \in \mathbb{R}^{2 d}$,

$$
\left|\operatorname{det}\left(\varphi_{x \xi}^{\prime \prime}\right)(x, \xi)\right| \geq r .
$$

Theorem 33 shows that the composition of SG FIOs of type I and type II with the same regular SG phase functions is a SG pseudodifferential operator, see [5] for a detailed proof.

Theorem 33. Let $\varphi \in \mathcal{P}_{r}$ and assume $a \in \mathrm{SG}^{m_{\psi}, m_{e}}\left(\mathbb{R}^{2 d}\right), b \in \mathrm{SG}^{s_{\psi}, s_{e}}\left(\mathbb{R}^{2 d}\right)$. Then,

$$
\begin{aligned}
& \mathrm{Op}_{\varphi}(a) \circ \mathrm{Op}_{\varphi}^{*}(b)=\mathrm{Op}\left(c_{5}+r_{5}\right)=\mathrm{Op}\left(c_{5}\right) \quad \bmod \mathrm{OpSG} \mathrm{SG}^{-\infty,-\infty}\left(\mathbb{R}^{d}\right), \\
& \mathrm{Op}_{\varphi}^{*}(b) \circ \mathrm{Op}_{\varphi}(a)=\mathrm{Op}\left(c_{6}+r_{6}\right)=\mathrm{Op}\left(c_{6}\right) \bmod \mathrm{OpSG} \mathrm{SG}^{-\infty,-\infty}\left(\mathbb{R}^{d}\right) \text {, }
\end{aligned}
$$

for some $c_{j} \in \mathrm{SG}^{m_{\psi}+s_{\psi}, t_{\psi}+t_{e}}\left(\mathbb{R}^{2 d}\right), r_{j} \in \mathrm{SG}^{-\infty,-\infty}\left(\mathbb{R}^{2 d}\right), j=5,6$.

Furthermore, asymptotic formulae can be given for $c_{j}, j=1, \ldots, 6$, in terms of $\varphi, p, a$ and $b$, see [5].

Remark 34. In particular, we have the following first-order expansion of the symbol of $c_{1}$, coming from [5]:

$$
c_{1}(x, \xi)=p\left(x, \varphi_{x}^{\prime}(x, \xi)\right) a(x, \xi)+s(x, \xi), \quad s \in \mathrm{SG}^{m_{\psi}+s_{\psi}-1, m_{e}+t_{e}-1}\left(\mathbb{R}^{2 d}\right) .
$$

Remark 35. All the results in this section have classical counterparts, that is, when all the starting symbols and phase functions are SG-classical, the resulting objects are SG-classical as well, see [11].

Given a symbol $p \in \mathrm{SG}_{\mathrm{cl}}^{1,1}$, let us consider the eikonal equation

$$
\left\{\begin{aligned}
\partial_{t} \varphi(t, x, \xi)+p\left(x, \varphi_{x}^{\prime}(t, x, \xi)\right) & =0, \quad t \in[0, T] \\
\varphi(0, x, \xi) & =x \xi
\end{aligned}\right.
$$

By the theory developed in [6,11], Proposition 36 holds true.

Proposition 36. For some small enough $T_{0} \in(0, T]$, Eq. (26) admits a unique solution $\varphi \in C^{1}\left(\left[0, T_{0}\right], \mathrm{SG}_{\mathrm{cl}}^{1,1}\left(\mathbb{R}^{2 d}\right)\right)$. Moreover, $\varphi(t, x, \xi) \in \mathcal{P}_{r}$ for all $0 \leq t \leq$ $T_{0}$.

Remark 3\%. Using the standard procedure for solving hyperbolic evolution equations modulo regular terms (cf. $[6,10,11]$ ), it is possible to construct a short-time parametrix for $\mathrm{e}^{-i t P}$, where $P \in \mathrm{OpSG}_{\mathrm{cl}}^{1,1}$. 


\section{A.3. Trace Operators on the SG-Algebra}

Various trace functionals on $\mathrm{OpSG}_{\mathrm{cl}}\left(\mathbb{R}^{d}\right)$ were introduced and studied by Nicola in [31], including a notion of Wodzicki residue. In [3], such concepts have been extended to SG-classical operators on the class of manifolds with ends described in Section A.4, see also [23,24]. Here, we recall the definitions of such functionals in terms of the symbolic structure, as well as their relation with the spectral $\zeta$-function.

Let $A \in \mathrm{OpSG}_{\mathrm{cl}}^{m_{\psi}, m_{e}}\left(\mathbb{R}^{d}\right)$ be elliptic, self-adjoint, and positive. ${ }^{5}$ We set

$$
\begin{aligned}
\operatorname{Tr}_{\psi, e}(A)= & \frac{1}{(2 \pi)^{d}} \int_{\mathbb{S}^{d-1}} \int_{\mathbb{S}^{d-1}} a_{-d,-d}\left(\theta, \theta^{\prime}\right) \mathrm{d} \theta^{\prime} \mathrm{d} \theta=\frac{1}{(2 \pi)^{d}} I_{-d}^{-d}, \\
\widehat{\operatorname{Tr}}_{\psi}(A)= & \frac{1}{(2 \pi)^{d}} \lim _{\tau \rightarrow \infty}\left[\int_{|x| \leq \tau} \int_{\mathbb{S}^{d-1}} a_{-d, \cdot}(x, \theta) \mathrm{d} \theta \mathrm{d} x\right. \\
& \left.-(\log \tau) I_{-d}^{-d}-\sum_{k=0}^{m_{e}+d-1} \frac{\tau^{m_{e}-k}}{\left(m_{e}-k\right)} I_{-d}^{m_{e}-k}\right], \\
\widehat{\operatorname{Tr}}_{e}(A)= & \frac{1}{(2 \pi)^{d}} \lim _{\tau \rightarrow \infty}\left[\int_{\mathbb{S}^{d-1}} \int_{|\xi| \leq \tau} a_{\cdot,-d}(\theta, \xi) \mathrm{d} \xi \mathrm{d} \theta\right. \\
& \left.-(\log \tau) I_{-d}^{-d}-\sum_{j=0}^{m_{\psi}+d-1} \frac{\tau^{m_{\psi}-j}}{\left(m_{\psi}-j\right)} I_{m_{\psi}-j}^{-d}\right]
\end{aligned}
$$

where, respectively,

$$
\begin{aligned}
& I_{-d}^{m_{e}-k}=\int_{\mathbb{S}^{d-1}} \int_{\mathbb{S}^{d-1}} a_{-d, m_{e}-k}\left(\theta^{\prime}, \theta\right) \mathrm{d} \theta \mathrm{d} \theta^{\prime}, \\
& I_{m_{\psi}-j}^{-d}=\int_{\mathbb{S}^{d-1}} \int_{\mathbb{S}^{d-1}} a_{m_{\psi}-j,-d}\left(\theta^{\prime}, \theta\right) \mathrm{d} \theta \mathrm{d} \theta^{\prime} .
\end{aligned}
$$

A further functional $\widehat{\mathrm{TR}}_{\theta}(A)$ was defined in $[3,(3.6)]$, called angular term:

$$
\widehat{\mathrm{TR}}_{\theta}(A)=\frac{1}{(2 \pi)^{n}} \int_{\mathbb{S}^{n-1}} \int_{\mathbb{S}^{n-1}}\left[\frac{d}{\mathrm{~d} z} a_{m_{\psi} z-d-m_{\psi}, m_{e} z-d-m_{e}}(z)\right]_{z=1}\left(\theta, \theta^{\prime}\right) \mathrm{d} \theta^{\prime} \mathrm{d} \theta .
$$

Remark 38. In general, it is rather cumbersome to evaluate the angular term defined in (27). In the case $m_{\psi}=m_{e}=-d$, the computation is easier. Indeed, by [3, Proposition 1.10],

$$
\left.\frac{d}{\mathrm{~d} z} a_{-d z,-d z}(z)\right|_{z=1}=\lim _{z \rightarrow 1} \frac{a_{-d z,-d z}(z)-a_{-d,-d}}{z-1}=a_{-d,-d} \cdot \log \left(a_{-d,-d}\right) .
$$

One can also define the traces as the residues of the spectral zeta function (cf. [3, Section 3 and Section 4]). Notice the opposite sign convention for $\zeta(s)=$ $\operatorname{Tr} A^{-s}$ in [3].

\footnotetext{
${ }^{5}$ As explained in [3], it suffices to assume $\Lambda$-ellipticity and some sectoriality conditions on the spectrum.
} 
Definition 39. The trace operators $\mathrm{TR}$ and $\widehat{\mathrm{TR}}_{x, \xi}$ are given by

$$
\begin{aligned}
\operatorname{TR}(A) & =m_{\psi} m_{e} \operatorname{Res}_{s=-1}^{2} \zeta(s)=m_{\psi} m_{e} \lim _{s \rightarrow-1}(s+1)^{2} \zeta(s), \\
\widehat{\operatorname{TR}}_{x, \xi}(A) & =\lim _{s \rightarrow-1}(s+1)\left[\zeta(s)-\frac{\operatorname{Res}_{s=-1}^{2} \zeta(s)}{(s+1)^{2}}\right] .
\end{aligned}
$$

The following proposition was proved in [3, Theorem 3.3 and Theorem 3.4] with arguments similar to the one employed in the proof of Proposition 19. It gives the relation between the functionals $\operatorname{Tr}_{\psi, e}(A), \widehat{\operatorname{Tr}}_{\psi}(A), \widehat{\operatorname{Tr}}_{e}(A)$, $\widehat{\mathrm{TR}}_{\theta}(A)$, and the spectral $\zeta$-function of $A$.

Proposition 40. Let $A$ be as above. Then,

$$
\begin{aligned}
\operatorname{TR}(A) & =\operatorname{Tr}_{\psi, e}(A), \\
\widehat{\operatorname{TR}}_{x, \xi}(A) & =-\frac{1}{m_{\psi}} \widehat{\operatorname{Tr}}_{\psi}(A)-\frac{1}{m_{e}} \widehat{\operatorname{Tr}}_{e}(A)+\frac{1}{m_{\psi} m_{e}} \widehat{\operatorname{TR}}_{\theta}(A) .
\end{aligned}
$$

The functional TR, the Wodzicki residue for the SG-classical operators setting (cf., e.g. [3,31] and the references therein), can be extended to all SGclassical operators with integer order in a standard way, cf. [21]. It is also possible to prove that $\mathrm{TR}$ is a trace on the algebra $\mathscr{A}=\mathrm{OpSG}_{\mathrm{cl}} / \mathrm{OpSG}^{-\infty,-\infty}$, see again [21].

\section{A.4. Manifolds with Cylindrical Ends}

We briefly recall the definition of a class of manifolds with cylindrical ends given in [3], together with the concepts, in such environment, of rapidly decreasing function, temperate distribution, SG-calculus, and weighted Sobolev space. In [3], such notions have been illustrated with slight modifications with respect to their original definition in [22]. This class of manifolds was introduced by describing explicitly the admissible atlases, in the spirit of the definition of SG-manifolds given by Schrohe [34]. Without loss of generality, to keep notation simpler, we focus on the manifolds with a single cylindrical end.

Definition 41. A manifold with a cylindrical end of dimension $d$ is a triple $(M, Y,[f])$, where $M=\mathscr{M} \amalg_{C} \mathscr{C}$ is a $d$-dimensional smooth manifold and

1. $\mathscr{M}$ is a smooth manifold, given by $\mathscr{M}=\left(M_{0} \backslash D\right) \cup C$ with a $d$-dimensional smooth compact manifold without boundary $M_{0}, D$ a closed disc of $M_{0}$, and $C \subset D$ a collar neighbourhood of $\partial D$ in $M_{0}$;

2. $\mathscr{C}$ is a smooth manifold with boundary $\partial \mathscr{C}=Y$, with $Y$ diffeomorphic to $\partial D$;

3. $f:\left[\delta_{f}, \infty\right) \times \mathbb{S}^{d-1} \rightarrow \mathscr{C}, \delta_{f}>0$, is a diffeomorphism, $f\left(\left\{\delta_{f}\right\} \times \mathbb{S}^{d-1}\right)=Y$, and $f\left(\left[\delta_{f}, \delta_{f}+\varepsilon_{f}\right) \times \mathbb{S}^{d-1}\right), \varepsilon_{f}>0$, is diffeomorphic to $C$;

4. the symbol $\amalg_{C}$ means that we are gluing $\mathscr{M}$ and $\mathscr{C}$, through the identification of $C$ and $f\left(\left[\delta_{f}, \delta_{f}+\varepsilon_{f}\right) \times \mathbb{S}^{d-1}\right)$; 
5. the symbol $[f]$ represents an equivalence class in the set of functions $\left\{g:\left[\delta_{g}, \infty\right) \times \mathbb{S}^{d-1} \rightarrow \mathscr{C}: g\right.$ is a diffeomorphism,

$$
\begin{aligned}
& g\left(\left\{\delta_{g}\right\} \times \mathbb{S}^{d-1}\right)=Y \text { and } \\
& \left.g\left(\left[\delta_{g}, \delta_{g}+\varepsilon_{g}\right) \times \mathbb{S}^{d-1}\right), \varepsilon_{g}>0, \text { is diffeomorphic to } C\right\}
\end{aligned}
$$

where $f \sim g$ if and only if there exists a diffeomorphism $\Theta \in \operatorname{Diff}\left(\mathbb{S}^{d-1}\right)$ such that

$$
\left(g^{-1} \circ f\right)(\rho, \omega)=(\rho, \Theta(\omega))
$$

for all $\rho \geq \max \left\{\delta_{f}, \delta_{g}\right\}$ and $\omega \in \mathbb{S}^{d-1}$.

We use the following notation:

- $U_{\delta_{f}}=\left\{x \in \mathbb{R}^{d}:|x|>\delta_{f}\right\}$;

- $\mathscr{C}_{\tau}=f\left([\tau, \infty) \times \mathbb{S}^{d-1}\right)$, where $\tau \geq \delta_{f}$. The equivalence condition (32) implies that $\mathscr{C}_{\tau}$ is well defined;

- $\pi: \mathbb{R}^{d} \backslash\{0\} \rightarrow(0, \infty) \times \mathbb{S}^{d-1}: x \mapsto \pi(x)=\left(|x|, \frac{x}{|x|}\right)$;

- $f_{\pi}=f \circ \pi: \overline{U_{\delta_{f}}} \rightarrow \mathscr{C}$ is a parameterization of the end. Let us notice that, setting $F=g_{\pi}^{-1} \circ f_{\pi}$, the equivalence condition (32) implies

$$
F(x)=|x| \Theta\left(\frac{x}{|x|}\right) .
$$

We also denote the restriction of $f_{\pi}$ mapping $U_{\delta_{f}}$ onto $\dot{\mathscr{C}}=\mathscr{C} \backslash Y$ by $\dot{f}_{\pi}$. The couple $\left(\dot{\mathscr{C}}, \dot{f}_{\pi}^{-1}\right)$ is called the exit chart. If $\mathscr{A}=\left\{\left(\Omega_{j}, \psi_{j}\right)\right\}_{j=1}^{N}$ is such that the subset $\left\{\left(\Omega_{j}, \psi_{j}\right)\right\}_{j=1}^{N-1}$ is a finite atlas for $\mathscr{M}$ and $\left(\Omega_{N}, \psi_{N}\right)=\left(\dot{\mathscr{C}}, \dot{f}_{\pi}^{-1}\right)$, then $M$, with the atlas $\mathscr{A}$, is a SG-manifold (see [34]). An atlas $\mathscr{A}$ of such kind is called admissible. From now on, we restrict the choice of atlases on $M$ to the class of admissible ones. We introduce the following spaces, endowed with their natural topologies:

$$
\begin{aligned}
& \mathscr{S}\left(U_{\delta}\right)=\left\{u \in \mathcal{C}^{\infty}\left(U_{\delta}\right): \forall \alpha, \beta \in \mathbb{N}^{n} \forall \delta^{\prime}>\delta \sup _{x \in U_{\delta^{\prime}}}\left|x^{\alpha} \partial^{\beta} u(x)\right|<\infty\right\}, \\
& \mathscr{S}_{0}\left(U_{\delta}\right)=\bigcap_{\delta^{\prime}}\left\{u \in \mathscr{S}\left(\mathbb{R}^{d}\right): \operatorname{supp} u \subseteq \overline{U_{\delta^{\prime}}}\right\}, \\
& \mathscr{S}(M)=\left\{u \in C^{\infty}(M): u \circ \dot{f}_{\pi} \in \mathscr{S}\left(U_{\delta_{f}}\right) \text { for any exit map } f_{\pi}\right\}, \\
& \mathscr{S}^{\prime}(M) \text { denotes the dual space of } \mathscr{S}(M) .
\end{aligned}
$$

$M$ is also tacitly assumed to be endowed with a volume form $\mathrm{d} \mu$ (for instance, induced by a Riemannian metric), so that the spaces $L^{p}(M), p \in[1, \infty]$, can be defined as well.

Definition 42. The set $\mathrm{SG}^{m_{\psi}, m_{e}}\left(U_{\delta_{f}}\right)$ consists of all the symbols $a \in \mathcal{C}^{\infty}\left(U_{\delta_{f}}\right)$ which fulfill $(5)$ for $(x, \xi) \in U_{\delta_{f}} \times \mathbb{R}^{d}$ only. Moreover, the symbol $a$ belongs to the subset $\mathrm{SG}_{\mathrm{cl}}^{m_{\psi}, m_{e}}\left(U_{\delta_{f}}\right)$ if it admits expansions in asymptotic sums of homogeneous symbols with respect to $x$ and $\xi$ as in Definition 9 , where the remainders are now given by SG-symbols of the required order on $U_{\delta_{f}}$. 
Note that since $U_{\delta_{f}}$ is conical, the definition of homogeneous and classical symbol on $U_{\delta_{f}}$ makes sense. Moreover, the elements of the asymptotic expansions of the classical symbols can be extended by homogeneity to smooth functions on $\mathbb{R}^{d} \backslash\{0\}$, which will be denoted by the same symbols. It is a fact that, given an admissible atlas $\left\{\left(\Omega_{j}, \psi_{j}\right)\right\}_{j=1}^{N}$ on $M$, there exists a partition of unity $\left\{\varphi_{j}\right\}$ and a set of smooth functions $\left\{\chi_{j}\right\}$ which are compatible with the SG-structure of $M$, that is (see [34]):

- $\operatorname{supp} \varphi_{j} \subset \Omega_{j}, \operatorname{supp} \chi_{j} \subset \Omega_{j}, \chi_{j} \varphi_{j}=\varphi_{j}, j=1, \ldots, N$;

- $\left|\partial^{\alpha}\left(\varphi_{N} \circ \dot{f}_{\pi}\right)(x)\right| \leq C_{\alpha}|x|^{-|\alpha|}$ and $\left|\partial^{\alpha}\left(\chi_{N} \circ \dot{f}_{\pi}\right)(x)\right| \leq C_{\alpha}|x|^{-|\alpha|}$ for all $x \in U_{\delta_{f}}$.

Moreover, $\varphi_{N}$ and $\chi_{N}$ can be chosen so that $\varphi_{N} \circ \dot{f}_{\pi}$ and $\chi_{N} \circ \dot{f}_{\pi}$ are homogeneous of degree 0 on $U_{\delta}$. We denote by $u^{*}$ the composition of $u: \psi_{j}\left(\Omega_{j}\right) \subset \mathbb{R}^{d} \rightarrow \mathbb{C}$ with the coordinate patches $\psi_{j}$, and by $v_{*}$ the composition of $v: \Omega_{i} \subset M \rightarrow \mathbb{C}$ with $\psi_{j}^{-1}, j=1, \ldots, N$. We now recall the definition of SG-pseudodifferential operator on $M$.

Definition 43. Let $M$ be a manifold with a cylindrical end. A linear operator $A: \mathscr{S}(M) \rightarrow \mathscr{S}^{\prime}(M)$ is a SG-pseudodifferential operator of order $\left(m_{\psi}, m_{e}\right)$ on $M$, and we write $A \in \mathrm{OpSG}^{m_{\psi}, m_{e}}(M)$ if, for any admissible atlas $\left\{\left(\Omega_{j}, \psi_{j}\right)\right\}_{j=1}^{N}$ on $M$ with exit chart $\left(\Omega_{N}, \psi_{N}\right)$ :

1. for all $j=1, \ldots, N-1$ and any $\varphi_{j}, \chi_{j} \in \mathcal{C}_{c}^{\infty}\left(\Omega_{j}\right)$, there exist symbols $a_{j} \in S^{m_{\psi}}\left(\psi_{j}\left(\Omega_{j}\right)\right)$ such that

$$
\begin{aligned}
& \left(\chi_{j} A \varphi_{j} u^{*}\right)_{*}(x) \\
& \quad=(2 \pi)^{-d} \iint \mathrm{e}^{i(x-y) \xi} \chi_{j}(x) a_{j}(x, \xi) \varphi_{j}(y) u(y) \mathrm{d} y \mathrm{~d} \xi, u \in \mathcal{C}^{\infty}\left(\psi_{j}\left(\Omega_{j}\right)\right) ;
\end{aligned}
$$

2. for any $\varphi_{N}, \chi_{N}$ of the type described above, there exists a symbol $a_{N} \in$ $\mathrm{SG}^{m_{\psi}, m_{e}}\left(U_{\delta_{f}}\right)$ such that

$$
\begin{aligned}
& \left(\chi_{N} A \varphi_{N} u^{*}\right)_{*}(x) \\
& \quad=(2 \pi)^{-d} \iint \mathrm{e}^{i(x-y) \xi} \chi_{N}(x) a_{N}(x, \xi) \varphi_{N}(y) u(y) \mathrm{d} y \mathrm{~d} \xi, u \in \mathscr{S}_{0}\left(U_{\delta_{f}}\right) ;
\end{aligned}
$$

3. $K_{A}$, the Schwartz kernel of $A$, is such that

$$
K_{A} \in \mathcal{C}^{\infty}((M \times M) \backslash \Delta) \bigcap \mathscr{S}((\dot{\mathscr{C}} \times \dot{\mathscr{C}}) \backslash W)
$$

where $\Delta$ is the diagonal of $M \times M$ and $W=\left(\dot{f}_{\pi} \times \dot{f}_{\pi}\right)(V)$ with any conical neighbourhood $V$ of the diagonal of $U_{\delta_{f}} \times U_{\delta_{f}}$.

The most important local symbol of $A$ is $a_{N}$, which we will also denote $a^{f}$, to remind its dependence on the exit chart. Our definition of SG-classical operator on $M$ differs slightly from the one in [22].

Definition 44. Let $A \in \mathrm{OpSG}^{m_{\psi}, m_{e}}(M)$. $A$ is a SG-classical operator on $M$, and we write $A \in \mathrm{OpSG}_{\mathrm{cl}}^{m_{\psi}, m_{e}}(M)$, if $a^{f}(x, \xi) \in \mathrm{SG}_{\mathrm{cl}}^{m_{\psi}, m_{e}}\left(U_{\delta_{f}}\right)$ and the operator $A$, restricted to the manifold $\mathscr{M}$, is classical in the usual sense. 
Remark 45. Since $M$ is a SG-manifold, the concepts of SG symbols and operators, as well as of the Schwartz spaces of functions and distributions, are invariant with respect to the choice of the atlas in the class of admissible ones. Given the special structure of the exit chart, the same is true also for the classical SG symbols and operators, see [22].

The principal homogeneous symbol $a_{m_{\psi}}$, of a SG-classical operator $A \in$ $\mathrm{OpSG}_{\mathrm{cl}}^{m_{\psi}, m_{e}}(M)$ is of course well defined as a smooth function on $T^{*} M \backslash 0$. In order to give an invariant definition of principal symbol with respect to $x$ of an operator $A \in \mathrm{OpSG} \mathrm{Sl}_{\mathrm{cl}}^{m_{\psi}, m_{e}}(M)$, the subbundle $T_{Y}^{*} M=\left\{(x, \xi) \in T^{*} M: x \in\right.$ $\left.Y, \xi \in T_{x}^{*} M\right\}$ was introduced. The notion of ellipticity can be extended to operators on $M$ as well.

Definition 46. Let $A \in \mathrm{OpSG}_{\mathrm{cl}}^{m_{\psi}, m_{e}}(M)$, and let us fix an exit map $f_{\pi}$. We can define local objects $a_{m_{\psi}-j, m_{e}-k}, a_{\cdot, m_{e}-k}$ as

$$
\begin{aligned}
a_{m_{\psi}-j, m_{e}-k}(\theta, \xi) & =a_{m_{\psi}-j, m_{e}-k}^{f}(\theta, \xi), \quad \theta \in \mathbb{S}^{d-1}, \xi \in \mathbb{R}^{d} \backslash\{0\}, \\
a_{\cdot, m_{e}-k}(\theta, \xi) & =a_{\cdot, m_{e}-k}^{f}(\theta, \xi), \quad \theta \in \mathbb{S}^{d-1}, \xi \in \mathbb{R}^{d} .
\end{aligned}
$$

Definition 47. An operator $A \in \mathrm{OpSG}_{\mathrm{cl}}^{m_{\psi}, m_{e}}(M)$ is elliptic if the principal part of $a^{f} \in S G^{m_{\psi}, m_{e}}\left(U_{\delta_{f}}\right)$ satisfies the SG-ellipticity conditions on $U_{\delta_{f}} \times \mathbb{R}^{d}$ and the operator $A$, restricted to the manifold $\mathscr{M}$, is elliptic in the usual sense.

Proposition 48. The properties $A \in \mathrm{OpSG}^{m_{\psi}, m_{e}}(M)$ and $A \in \mathrm{OpSG}_{\mathrm{cl}}^{m_{\psi}, m_{e}}(M)$ as well as the notions of ellipticity do not depend on the (admissible) atlas. Moreover, the local functions $a_{\cdot, m_{e}}$ and $a_{m_{\psi}, m_{e}}$ give rise to invariantly defined elements of $\mathcal{C}^{\infty}\left(T_{Y}^{*} M\right)$ and $\mathcal{C}^{\infty}\left(T_{Y}^{*} M \backslash 0\right)$, respectively.

Then, with any $A \in \mathrm{OpSG}_{\mathrm{cl}}^{m_{\psi}, m_{e}}(M)$, it is associated an invariantly defined principal symbol in three components $\sigma(A)=\left(a_{m_{\psi}, .}, a_{., m_{e}}, a_{m_{\psi}, m_{e}}\right)$. Finally, through local symbols given by $p_{j}(x, \xi)=\langle\xi\rangle^{s_{\psi}}, j=1, \ldots, N-1$, and $p^{f}(x, \xi)=\langle\xi\rangle^{s_{\psi}}\langle x\rangle^{s_{e}}, s_{\psi}, s_{e} \in \mathbb{R}$, we get a SG-elliptic operator $\Pi_{s_{\psi}, s_{e}} \in$ $\mathrm{OpSG}_{\mathrm{cl}}^{s_{\psi}, s_{e}}(M)$ and introduce the (invariantly defined) weighted Sobolev spaces $H^{s_{\psi}, s_{e}}(M)$ as

$$
H^{s_{\psi}, s_{e}}(M)=\left\{u \in \mathscr{S}^{\prime}(M): \Pi_{s_{\psi}, s_{e}} u \in L^{2}(M)\right\} .
$$

The properties of the spaces $H^{s_{\psi}, s_{e}}\left(\mathbb{R}^{d}\right)$ extend to $H^{s_{\psi}, s_{e}}(M)$ without any change, as well as the continuous action on them of the SG-operators.

Proposition 49. ([3])n The zeta function $\zeta(s)=\operatorname{Tr} A^{-s}$ and the trace operators $\operatorname{TR}(A)$ and $\widehat{\operatorname{TR}}_{x, \xi}(A)$ are well defined for any positive elliptic $A \in$ $\mathrm{OpSG}_{\mathrm{cl}}^{m_{\psi}, m_{e}}(M)$ on a manifold with cylindrical ends $M$.

The relationship between manifolds with cylindrical ends and asymptotically Euclidean manifolds is as follows. Let $M$ be a manifold with cylindrical ends as in Definition 41. Then, we can use the radial compactification and choose a scattering metric on the compactification $X$ that is compatible with the SG-structure (see the similar concept of $\mathcal{S}$-manifolds in [4, Sects. 4.1, 4.2]). 


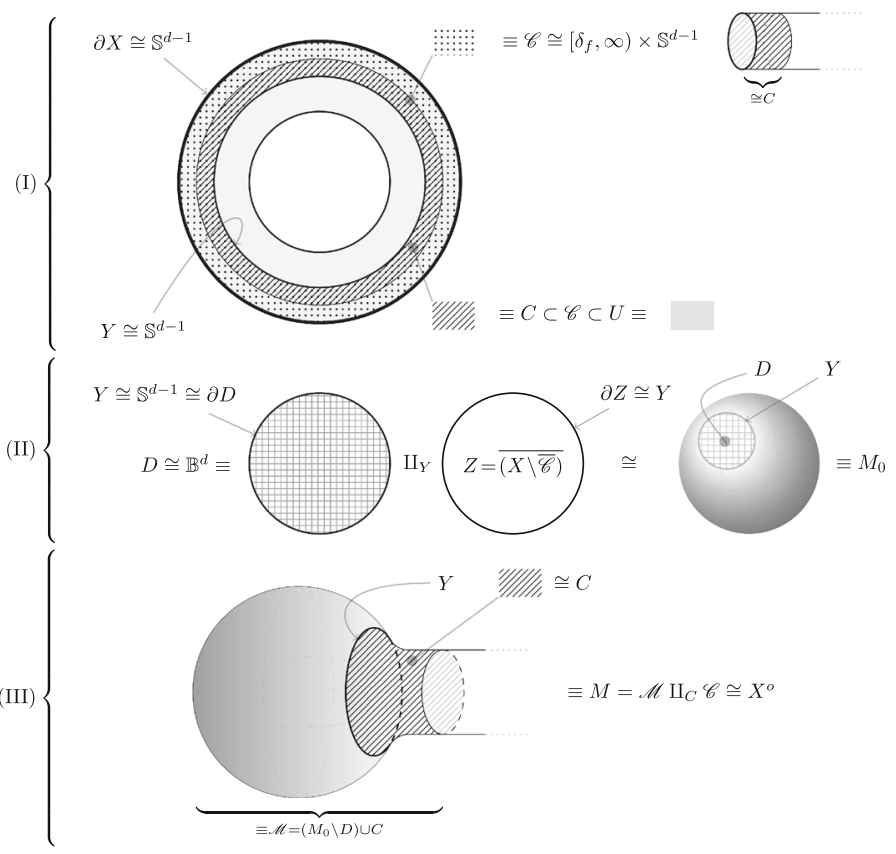

FiguRE 1. An asymptotically Euclidean manifold $X$ with $\partial X \cong \mathbb{S}^{d-1}$ as the compactification of a manifold $M$ with a cylindrical end. Step (I): decomposition of $X$. Step (II): construction of $M_{0}$. Step (III): construction of $M$

The boundary $\partial X$ is then a disjoint union of components diffeomorphic to spheres.

On the other hand, if the boundary of an asymptotically Euclidean manifold $(X, g)$, with boundary defining function $\rho_{X}$, consists of a disjoint union of components diffeomorphic to spheres, then it is the compactification of a manifold with cylindrical ends with the same SG-structure (see Fig. 1). In fact, we may assume without loss of generality that the boundary has a single connected component, that is, $\partial X \cong \mathbb{S}^{d-1}$. Choose $\delta>0$ sufficiently small, and let $U \subset X$ be a relatively open collar neighbourhood of $\partial X$, with coordinates $\phi:[0, \delta) \times \mathbb{S}^{d-1} \rightarrow U$, such that $\rho_{X}(\phi(r, \theta))=r$ for all $(r, \theta) \in[0, \delta) \times \mathbb{S}^{d-1}$ and $\rho_{X}(x) \geq \delta$ for $x \in X \backslash U$.

We set $M=X^{o}$ and $\mathscr{C}=\phi\left((0, \delta / 2] \times \mathbb{S}^{d-1}\right), C=\phi\left((\delta / 4, \delta / 2] \times \mathbb{S}^{d-1}\right)$, and define the local coordinates near the boundary

$$
\begin{aligned}
f:\left[\delta_{f}, \infty\right) \times \mathbb{S}^{d-1} & \rightarrow \mathscr{C} \\
(r, \theta) & \mapsto \phi(1 / r, \theta),
\end{aligned}
$$

where $\delta_{f}=2 / \delta$.

We find $Y=\partial \mathscr{C}=\left\{x \in M: \rho_{X}(x)=\delta / 2\right\} \cong \mathbb{S}^{d-1} \cong \partial X$. Finally, setting $D=\mathbb{B}^{d}, M_{0}=\overline{(X \backslash \overline{\mathscr{C}})} \amalg_{Y} D$, and $\mathscr{M}=\left(M_{0} \backslash D\right) \cup C$, we obtain a 
decomposition of $M$ as in Definition 41 and $f$ satisfies condition (3) of Definition 41 with $\varepsilon_{f}=2 / \delta$. Then, $(M, Y,[f])$ is a manifold with cylindrical ends. As in [7, Example 1.14], we see that $\operatorname{SG}^{m_{\psi}, m_{e}}(M) \cong \rho_{X}^{-m_{e}} \rho_{\Xi}^{-m_{\psi}} \mathcal{C}^{\infty}\left({ }^{\mathrm{sc}} \bar{T}^{*} X\right)$ under radial compactification, ${ }^{6}$ which proves the claim. In this sense, we may view manifolds with cylindrical ends as a proper subclass of asymptotically Euclidean manifolds.

To keep this exposition within a reasonable length, and avoid to deviate from our main focus, the detailed analysis of the extension of some of the existing results mentioned above, which we employ to prove the main theorems of this paper, to general asymptotically Euclidean manifolds will be illustrated elsewhere.

\section{References}

[1] Ascanelli, A., Coriasco, S.: Fourier integral operators algebra and fundamental solutions to hyperbolic systems with polynomially bounded coefficients on $\mathbb{R}^{n}$. J. Pseudo Differ. Oper. Appl. 6(4), 521-565 (2015)

[2] Battisti, U.: Weyl asymptotics of bisingular operators and Dirichlet divisor problem. Math. Z. 272(3), 1365-1381 (2012)

[3] Battisti, U., Coriasco, S.: Wodzicki residue for operators on manifolds with cylindrical ends. Ann. Global Anal. Geom. 40(2), 223-249 (2011)

[4] Cordes, H.O.: The Technique of Pseudodifferential Operators. Cambridge University Press, Cambridge (1995)

[5] Coriasco, S.: Fourier integral operators in SG classes. I. Composition theorems and action on SG Sobolev spaces. Rend. Sem. Mat. Univ. Politec. Torino 57(4), 249-302 (1999)

[6] Coriasco, S.: Fourier integral operators in SG classes. II. Application to SG hyperbolic Cauchy problems, English, with English and Italian summaries. Ann. Univ. Ferrara Sez. VII (N.S.) 44, 81-122 (1998)

[7] Coriasco, S., Doll, M., Schulz, R.: Lagrangian distributions on asymptotically Euclidean manifolds. Ann. Mat. Pura Appl. (4) 198(5), 1731-1780 (2019). issn:0373-3114

[8] Coriasco, S., Johansson, K., Toft, J.: Global wave-front properties for Fourier integral operators and hyperbolic problems. J. Fourier Anal. Appl. 22(2), 285333 (2016)

[9] Coriasco, S., Maniccia, L.: Wave front set at infinity and hyperbolic linear operators with multiple characteristics. Ann. Global Anal. Geom. 24(4), 375-400 (2003)

[10] Coriasco, S., Maniccia, L.: On the spectral asymptotics of operators on manifolds with ends. Abstr. Appl. Anal., Art. ID 909782, p. 21 (2013)

[11] Coriasco, S., Panarese, P.: Fourier integral operators defined by classical symbols with exit behaviour. Math. Nachr. 242, 61-78 (2002)

[12] Coriasco, S., Schulz, R.: The global wave front set of tempered oscillatory integrals with inhomogeneous phase functions. J. Fourier Anal. Appl. 19(5), 10931121 (2013)

\footnotetext{
${ }^{6}$ In the coordinates above, the radial compactification map is given by $(r, \theta) \mapsto(1 / r, \theta)$.
} 
[13] Coriasco, S., Schulz, R.: Lagrangian submanifolds at infinity and their parametrization. J. Symp. Geom. 15(4), 937-982 (2017)

[14] Doll, M., Gannot, O., Wunsch, J.: Refined Weyl law for homogeneous perturbations of the harmonic oscillator. Commun. Math. Phys. 362(1), 269-294 (2018)

[15] Duistermaat, J.J., Guillemin, V.W.: The spectrum of positive elliptic operators and periodic bicharacteristics. Invent. Math. 29(1), 39-79 (1975)

[16] Gradshteyn, I.S., Ryzhik, I.M.: Table of Integrals, Series, and Products, xv+1160. Academic Press, New York (1980), ISBN: 0-12-294760-6

[17] Grieser, D., Gruber, M.J.: Singular asymptotics lemma and push-forward theorem. In: Approaches to Singular Analysis (Berlin, 1999), Oper. Theory Adv. Appl., vol. 125, pp. 117-130. Birkhäuser, Basel (2001)

[18] Hörmander, L.: The spectral function of an elliptic operator. Acta Math. 121, 193-218 (1968)

[19] Hörmander, L.: The analysis of linear partial differential operators. III. Classics in Mathematics, Pseudo-differential operators; Reprint of the 1994 edition, Springer, Berlin, 2007, viii+525. ISBN: 978-3-540-49937-4

[20] Hörmander, L.: The analysis of linear partial differential operators. IV. Classics in Mathematics, Fourier integral operators; Reprint of the 1994 edition, Springer, Berlin (2009), viii+352. ISBN:978-3-642-00117-8

[21] Kassel, C.: Le résidu non commutatif (d'après M. Wodzicki), Séminaire Bourbaki, 1988,1989, Astérisque, 177-178, Exp. No. 708, 199-229 (1989)

[22] Maniccia, L., Panarese, P.: Eigenvalue asymptotics for a class of MD-elliptic $\psi$ do's on manifolds with cylindrical exits. Ann. Mat. Pura Appl. 181(3), 283$308(2002)$

[23] Maniccia, L., Schrohe, E., Seiler, J.: Complex powers of classical SGpseudodifferential operators. Ann. Univ. Ferrara 52, 353-369 (2006)

[24] Maniccia, L., Schrohe, E., Seiler, J.: Determinants of classical SGpseudodifferential operators. Math. Nachr. 287(7), 782-802 (2014)

[25] Melrose, R.B.: Calculus of conormal distributions on manifolds with corners. Int. Math. Res. Not. 3, 51-61 (1992)

[26] Melrose, R.B.: Geometric Scattering Theory, Stanford Lectures. Cambridge University Press, Cambridge (1995)

[27] Melrose, R.B.: Spectral and scattering theory for the Laplacianon asymptotically Euclidian spaces. In: Spectral and Scatteringtheory, Sanda, 1992, Lecture Notes in Pure and Appl. Math., vol. 161, pp. 85-130. Dekker, New York (1994)

[28] Melrose, R.B.: Differential analysis on manifolds with corners. Unpublished, partly available online at http://www-math.mit.edu/ rbm/book.html. Last downloaded 01/08/2016

[29] Melrose, R.B., Zworski, M.: Scattering metrics and geodesic flow at infinity. Invent. Math. 124(1-3), 389-436 (1996)

[30] Nicola, F., Rodino, L.: Global pseudo-differential calculus on Euclidean spaces. In: Pseudo-Differential Operators. Theory and Applications, vol. 4, pp. $\mathrm{x}+306$. Birkhäuser, Basel (2010). ISBN: 978-3-7643-8511-8

[31] Nicola, F.: Trace functionals for a class of pseudo-differential operators in $\mathbb{R}^{n}$. Math. Phys. Anal. Geom. 6(1), 89-105 (2003) 
[32] Parenti, C.: Operatori pseudodifferenziali in $\mathbb{R}^{n}$ e applicazioni. Ann. Mat. Pura Appl. 93, 359-389 (1972)

[33] Safarov, Yu., Vassiliev, D.: The asymptotic distribution of eigenvalues of partial differential operators. In: Translations of Mathematical Monographs, 155, Translated from the Russian Manuscript by the Authors, pp. xiv+354. American Mathematical Society, Providence (1997). ISBN: 0-8218-4577-2

[34] Schrohe, E.: Spaces of weighted symbols and weighted Sobolev spaces on manifolds. In: Oberwolfach (1986), Lecture Notes in Math., vol. 1256, pp. 360-377. Springer, Berlin (1987)

[35] Schrohe, E.: Complex powers on noncompact manifolds and manifolds with singularities. Math. Ann. 281(3), 393-409 (1988)

[36] Schrohe, E., Erkip, A.K.: Normal solvability of elliptic boundary value problems on asymptotically flat manifolds. J. Funct. Anal. 109(1), 22-51 (1992)

[37] Schulze, B.-W.: Boundary value problems and singular pseudo-differential operators. In: Pure and Applied Mathematics (New York), pp. xvi+637. Wiley, Chichester (1998). ISBN: 0-471-97557-5

[38] Shubin, M.A.: Pseudodifferential operators in $\mathbb{R}^{n}$. Sov. Math. Dokl. 12, 147-151 (1987)

[39] Wunsch, J.: The trace of the generalized harmonic oscillator, English, with English and French summaries. Ann. Inst. Fourier (Grenoble) 49(1), 351-373 (1999)

Sandro Coriasco

Dipartimento di Matematica "G. Peano"

Università degli Studi di Torino

V. C. Alberto, n. 10

10123 Turin

Italy

e-mail: sandro.coriasco@unito.it

Moritz Doll

Department 3 - Mathematics

University of Bremen

Bibliotheksstr. 5

28359 Bremen

Germany

e-mail: doll@uni-bremen.de

Communicated by Jan Derezinski.

Received: January 21, 2020.

Accepted: November 25, 2020. 